\title{
8 Funktionsverben und Funktionsverbgefüge im Sprachsystem
}

\subsection{Lexikoneinträge für FV}

Nachdem gezeigt werden konnte, dass FVG sich hinsichtlich der Selektion ihrer nominalen Bestandteile deutlich weniger arbiträr verhalten, als normalerweise angenommen, ist eine wichtige Grundlage für die weitere Einordnung des Phänomens FVG geschaffen. Im Anschluss an die Beobachtungen in den vorangegangenen Kapiteln ist nun die zentrale Frage zu klären, wie FVG bzw. FV repräsentiert sind: Ist für jedes FV ein Lexikoneintrag anzunehmen oder fällt der Lexikoneintrag des FV mit dem Eintrag für das jeweilige Vollverb zusammen? Ist überhaupt ein Eintrag für das FV vorzusehen? Wie ist das gesamte FVG zu modellieren? Muss für jedes einzelne FVG ein Eintrag vorgesehen werden? Sind nicht auch Generalisierungen denkbar, die einzelne FVG zu übergeordneten $\mathrm{Mu}$ stern zusammenfassen? Gibt es vielleicht sogar eine Regel, die FVG hervorbringt, so dass gar kein Lexikoneintrag für das Gefüge notwendig ist? Im Folgenden wird zunächst auf das FV eingegangen; im Anschluss daran wird der Status des FVG diskutiert.

\subsubsection{FV als reguläre, non-manner'-Verben}

Bei der Sichtung der verschiedenen Repräsentationsmöglichkeiten für das FV sei mit dem Vorschlag von Winhart (2005) zunächst eine vergleichsweise radikale Lösung diskutiert. Winhart zufolge besteht keine Notwendigkeit, für das FV oder FVG eine gesonderte Repräsentation anzunehmen. Sie plädiert stattdessen dafür, FVG als reguläre Verbindungen zu analysieren, die generellen grammatischen Prinzipien unterliegen. Was nach verbreiteter Auffassung für die Nominalisierungen in FVG gilt, nämlich dass für das Nomen in FVG kein eigener Ansatz nötig ist, nimmt Winhart auch für die Verben in Anspruch: Diese seien nicht desemantisiert, vielmehr besäßen sie die grammatischen Eigenschaften der Vollverben. Die Äußerungen Die Mutter gibt dem Kind einen Apfel und Die Mutter gibt dem Kind einen Rat unterschieden sich nicht prinzipiell, da in beiden der (implizite) Vorzustand die Negation des Nachzustands darstelle (Winhart 2005: 82f.). Die in FVG häufig auftretenden Verben kommen, bringen, geben und machen seien

Ә Open Access. () 2021 Volker Harm, publiziert von De Gruyter. (cc) BY Dieses Werk ist lizenziert unter der Creative Commons Attribution-NonCommercial-NoDerivatives 4.0 Lizenz.

https://doi.org/10.1515/9783110661255-008 
dadurch gekennzeichnet, dass es sich um „non-manner-Verben“ handele, $\mathrm{d}$. h. um Verben, die, anders als z. B. hämmern oder krabbeln, kein Argument in ihrer lexikalischen Dekomposition enthalten, das die Art und Weise der Handlungsausführung spezifiziert (vgl. Winhart 2005: 113). Non-manner-Verben seien aufgrund ihrer stark abstrakten Bedeutung besonders gut für die Kombination mit Ereignisnomina geeignet. ${ }^{180}$ Winhart geht daher davon aus, „dass es keine spezifischen Funktionsverben mit ,entleerter' Semantik gibt, sondern nur bestimmte Lesarten von Verben, die durch Übertragung eines z.B. lokalen Konzepts in ein temporales entstehen“ (2005: 75).

Ein Vorschlag wie der Winharts, der keine eigenen Lexikoneinträge für FV bzw. FVG vorsieht, ist attraktiv, weil er eine sehr ökonomische Beschreibung bietet. Dieser Ansatz lässt freilich Fragen offen. Wenn hier Vollverb und FV als vollkommen identisch angesehen werden, wird damit über deutlich sichtbare semantische Unterschiede hinweggegangen. Man wird jedenfalls kaum behaupten können, das das Verb in jmdn. zum Theater bringen und etwas zur Anwendung bringen semantisch vollständig übereinstimme. Ähnliches gilt auch für Winharts Beispiel geben: Wenn die Bedeutung des Verbs lediglich als Negation des Vorzustands durch den erreichten Nachzustand beschrieben wird, könnte man zwar in der Tat eine semantische Gemeinsamkeit zwischen jmdm. einen Rat geben und jmdm. einen Apfel geben postulieren. Diese Gemeinsamkeit ist freilich derart allgemein, dass sie kaum für den Ansatz eines einzigen Lexikoneintrags ausreicht, liegt doch bei allen Accomplishments im Prinzip eine Negation des Vorzustandes durch einen Nachzustand vor.

\subsubsection{FV als Realisierungen funktionaler Köpfe}

Die These, dass weder für das FV noch für das FVG als Ganzes ein eigener Lexikoneintrag vonnöten ist, wird auch in stark auf die Syntax fokussierten Beschreibungsansätzen vertreten. Dies gilt etwa für die in Kapitel 4 ausführlich erörterte Hypothese von Freeze (1992; 2001), der haben-Ausdrücke lediglich als lexikalische Ausbuchstabierungen zugrunde liegender syntaktischer Gegebenheiten

180 Eine generelle Annahme, dass non-manner-Verben abstrakter als andere Verben sind, kann jedoch in dieser Pauschalität nicht aufrecht erhalten werden. So zeichnen sich Verben, die in einen Resultatszustand münden (z. B. leeren, einfrieren, reinigen), grundsätzlich nicht durch ein manner-Argument aus; gleichwohl sind diese non-manner-Verben semantisch nicht als sonderlich abstrakt oder schematisch einzustufen; zum komplementären Verhältnis von „manner verb“ und „result verb“ s. Levin/Rappaport Hovav (1991: 147) und (2006: 1). 
analysiert hatte. Da haben Freeze (1992; 2001) zufolge keine lexikalische Kategorie bildet, wird in seinem Ansatz auch kein Lexikoneintrag vorgesehen. In eine ähnliche Richtung geht die Argumentation bei Businger (2011). Dieser führt haben letztlich auf eine präpositionale Komponente MIT zurück, die einen Small Clause einbettet (vgl. Businger 2011: 54; ähnlich auch Saebø 2009). Eine lexikalische Semantik sowie eine eigene Argumentstruktur wird dem Verb dabei explizit abgesprochen (Businger 2011: 266).

In vergleichbarer Weise werden bei Hale/Keyser (2002) Verben, die ,tun“/ ,machen' bedeuten, nicht von der lexikalischen Semantik, sondern von strukturellen Gegebenheiten her erklärt. Die genannten Verben seien, so die These, deshalb als ,light verbs‘ zu beschreiben, weil sie nicht intransitiv verwendet werden könnten, d. h. weil sie kein nicht-overtes Argument lizensieren, wie die Äußerungen in (1) belegen.
a. ${ }^{\star}$ He made.
b. ${ }^{\star}$ She did.

(vgl. Hale/Keyser 2002: 91)

Die Fähigkeit, ein nicht-overtes Argument zu lizensieren, wird in der Theorie von Hale/Keyser (2002) als grundlegend für unergative Verben (z. B. engl. sleep, dance, sneeze) erachtet. Diesen wird eine Struktur wie in (2) zugesprochen (hier am Beispiel engl. dance):

(2)

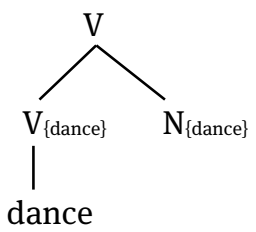

(Hale/Keyser 2002: 93)

Die Komponente $\{$ dance\}, die $\mathrm{V}$ und $\mathrm{N}$ gemeinsam haben, indiziert eine semantische Relation zwischen dem Verb und seinem Objekt. ,Light verbs‘ hingegen fehle gerade eine Struktur wie in (2); sie könnten daher auch kein non-overtes Objekt lizensieren, wie die ungrammatischen Beispiele (1) dieser These zufolge zeigen.

Während Hale/Keyser (2002: 93) den Verben engl. make und do offenbar jeden lexikalisch-semantischen Gehalt absprechen (,without any semantic component“), enthalte engl. put immerhin ,the idea of effecting a relation between two entities“ (2002: 94) als lexikalisch-semantische Komponente. Entscheidend für 
dessen Klassifikation als ,light verb‘ ist aber auch hier das mangelnde Vermögen, ein non-overtes Argument zu lizensieren, vgl. (3).

(3) *John put the books.

Die Argumentation von Hale/Keyser (2002) ist vor dem Hintergrund einer generellen Theorie der Argumentzuweisung zu sehen: In diesem Ansatz wird davon ausgegangen, dass der verbale Kopf unergativer Verben in der Phonologischen Form (PF) nicht interpretierbar sei und aus diesem Grund einen Prozess auslöse, den Hale/Keyser (2002: 63) als „,conflation“ bezeichnen: Im Zuge einer „conflation“ werde der phonologische Gehalt des non-overten Komplements in das leere V kopiert (,an empty head must always fuse with the head of its complement“ Hale/Keyser 2002: 161f.). Fehlt ein solches Komplement, ist dieser Prozess nicht möglich, weshalb (1) und (3) dieser These zufolge ungrammatisch sein müssen.

Ohne dass hier auf die Argumentation der Autoren im Einzelnen eingegangen werden kann, sei zumindest darauf hingewiesen, dass die Verben dt. kommen und geben durchaus absolut gebraucht werden können, vgl. (4).

a. Sie kam.

b. Wir nehmen nicht nur, wir geben.

Für diese Verben, die ja ebenfalls als FV auftreten, würde die Erklärung von Hale/Keyser (2002) daher nicht greifen bzw. es müsste in diesem Fall doch ein voll spezifizierter lexikalischer Kopf angenommen werden. FV aber teils als leere Köpfe (bei machen), teils als vollgültige lexikalische Einheiten (bei kommen und geben) zu analysieren, ist wenig attraktiv.

Grundsätzlich ist auch eine gewisse Zirkularität in der Argumentation unverkennbar: Einerseits wird argumentiert, dass make deshalb semantisch leer ist, weil es keine Struktur mit Komplement wie in (2) aufweist (Hale/Keyser 2002: 91), andererseits heißt bei Hale/Keyser (2002: 94), dass das Verb keinen ausreichenden semantischen Gehalt besitze, um ein verdecktes Komplement zu lizensieren (,[...] too ,light“ to license a nonovert argument“). Das Fehlen einer Struktur wie in (2) wird also letztlich doch mit semantischen Eigenschaften des Verbs begründet, d. h. konkret mit seiner fehlenden Semantik.

Eine Rückführung von FV auf syntaktische Gegebenheiten, wie sie in dem Ansatz von Hale/Keyser (2002) versucht wird, wirft weitere grundsätzliche Probleme auf. So bleibt unklar, wie Gebrauchsrestriktionen plausibel gemacht werden können, wenn man kein semantisch voll spezifiziertes Verb annimmt. Weiterhin müsste in einem solchen Ansatz demonstriert werden, in welcher Weise 
das breit ausgebaute Spektrum lexikalischer Bedeutungen, welches gerade Verben wie haben, geben, machen usw. zeigen, auf einer nicht-lexikalischen Basis aufsetzen kann. Für jede Lesart im polysemen Spektrum müsste eine eigene syntaktische Grundkonstellation nachgewiesen werden, was die syntaktische Komponente außerordentlich stark belasten würde. Aufgrund dieser Schwierigkeiten wird dieser Erklärungstyp hier nicht weiter verfolgt.

\subsubsection{FV und Vollverb in Modellierungen der HPSG}

Im Gegensatz zu den eben erörterten Hypothesen, die einen eigenen Lexikoneintrag für FV ablehnen, werden in Ansätzen, die sich im Rahmen der Head Driven Phrase Structure Grammar (HPSG) bewegen, eigene Lexikoneinträge für FV postuliert. Gute Argumente dafür, dass FV einen eigenen Lexikoneintrag erhalten sollten, der vom Lexikoneintrag für das Vollverb zu unterscheiden ist, hat Sailer (2003: 261f.) in Bezug auf das Englische vorgebracht. Dass ein Kontrast zwischen dem FV und dem entsprechenden Vollverb besteht, lässt sich Sailer zufolge anhand der Bildbarkeit eines Gerundiums nachweisen:

(5) a. *The making of progress/headway is a precondition for success.

b. The making of cars is an interesting topic for a documentary.

(Sailer 2003: 261)

Im Deutschen sind die Möglichkeiten der Gerundiumsbildung und der Nominalisierung deutlich eingeschränkter. So lässt sich gerade für die häufigen FV ( $m a$ chen, kommen, bringen, haben, geben) keine dem Englischen vergleichbare Form erzeugen. Konversionsbildungen im Deutschen lassen aber immerhin insofern partiell vergleichbare Schlüsse zu, als z. B. machen als nominalisiertes FV in (6a) klar ungrammatisch ist, während die Nominalisierung des Vollverbs machen in (6b) in erster Linie wohl kein grammatisches, sondern ein stilistisches Problem ist (wegen des Vorhandensein besserer Alternativen wie z. B. Formen oder Herstellung).

(6) a. `Das Machen von ersten Schritten ist für jedes Kind ein besonderes Erlebnis.

b. 'Das Machen von Teigkringeln ist nicht schwer. 
Im Gegensatz zu den Konversionsbildungen, die für das Deutsche deutlich weniger klare Hinweise liefern als für das Englische, ergibt sich aber aus den idiosynkratischen Selektionseigenschaften von FV und Vollverb ein gutes Unterscheidungskriterium (vgl. Sailer 2003: 264). Aufgrund ihrer je eigenen Argumentselektion können so etwa für treffen die zwei Ansätze in (7) identifiziert werden.
a. treffen $_{\text {vollverb: }}<$ Zielscheibe, Punkt, ...>
b. treffen $_{\mathrm{Fv}}:<$ Entscheidung $>$

Ein konkreter Vorschlag, wie Lexikoneinträge für FV formuliert werden können, findet sich in dem HPSG-basierten Ansatz von Krenn/Erbach (1994). Die Autoren gehen davon aus, dass das FV, obwohl es den Kopf der Phrase bildet, semantisch leer ist. Sie unterscheiden demnach auch jeweils zwischen einem Lexikoneintrag für das Vollverb und für das FV. Als Beispiel wählen sie u. a. haben: Während das Vollverb über eine komplette Füllung des CONTENT-Attributs verfügt, enthält die entsprechende Position beim FV lediglich eine Angabe zur Aktionsart (Krenn/Erbach 1994: 389). Das FVG wird dann als Ergebnis einer Arbeitsteilung zwischen FV und Nomen verstanden: ,[...] the semantic relation introduced by the predicative noun is inherited to the support verb; the Aktionsart of the entire verb construction is specified by the support verb" (ebd.). Das eigentliche semantisch leere FV wird somit im Zuge eines Vererbungsprozesses gewissermaßen mit inhaltsseitiger Information aufgeladen, im Beispiel Angst haben erhält es die Bedeutung ,sich fürchten“ (in ähnlicher Weise auch Kay/Sag 2015: 18 zu nicht-dekomponierbaren Idiomen). Im Rahmen der HPSG ist diese Modellierung folgerichtig, da der Kopf die Eigenschaften der gesamten Phrase determiniert. Die These, dass das Verb haben im FVG die Bedeutung ,sich fürchten' annimmt und Angst, da es seine Inhaltsmerkmale an den Kopf überträgt, dann semantisch leer wäre, entspricht gleichwohl nicht der Intuition.

Auch das Problem der Selektionseigenschaften des FV ist hier noch nicht befriedigend gelöst. Krenn/Erbach (1994) können lediglich Listen von in Frage kommenden Nomina postulieren; wie diese Listen zustande kommen, wird nicht im Rahmen der HPSG modelliert. Krenn/Erbach (1994) deuten hier freilich einen interessanten Lösungsweg an:

At a deeper level of analysis, the lexical semantic information of the support verb plays a role in establishing the relationship between the argument structure of the predicative noun and that of the support verb. For example, in the support verb construction Angst haben, 
the mental state Angst is something that can be possessed in a metaphorical sense, and can therefore appear as the object of haben.

Die Annahme, dass eine spezifische Relation zwischen Nomen und Verb besteht, welche die Selektion steuert, erscheint mit dem Befund in Kapitel 5 kompatibel. ${ }^{181}$ Allerdings ist die Position von Krenn/Erbach (1994) insgesamt nicht widerspruchsfrei: Einerseits wird explizit ein semantisch leeres FV postuliert, andererseits wird dem FV in dem oben angeführten Zitat ein lexikalischer Gehalt zugesprochen („lexical semantic information of the support verb“), damit die Relation zwischen Verb und Nomen beschrieben werden kann. Ein vergleichbarer Widerspruch besteht, wie in Kapitel 5.6 angedeutet, auch für den hier vertretenen Ansatz, der ja ebenfalls von einem semantisch leeren Verb ausgeht, welches aber zugleich die Selektion der Nomina bestimmt. Um die Klärung dieses Widerspruchs wird es weiter unten gehen; hier sind zunächst noch weitere einschlägige Forschungspositionen zu erörtern.

\subsubsection{FV als Realisierungen eines verbalen ,Passepartouts' (Butt 2010)}

Die Überzeugung, dass es sich bei FV um eine grundsätzlich lexikalische Erscheinung und nicht um eine funktionale Katerogie handelt, wird auch im Ansatz von Butt (2010) vertreten (vgl. auch Butt/Geuder 2001 und Butt/Lahiri 2013). Im Gegensatz zu Sailer (2003), der separate Einträge für das Vollverb und das FV annimmt, geht Butt (2010) von einem einzigen Lexikoneintrag aus, der sowohl dem FV - hier der englischsprachigen Forschungstradition entsprechend als ,light verb' bezeichnet - und dem Vollverb (,main verb') zugrunde liegt. ,Light verbs‘ sind Butt zufolge zunächst dadurch definiert, dass sie gemeinsam mit einem anderen Prädikatsausdruck in einem Satz vorkommen. Sie haben die Funktion, diesen Prädikatsausdruck zu modifizieren und zu nuancieren; eine eigenständige Prädikation drücken sie nicht aus (Butt 2010: 74f.; Butt/Lahiri 2013: 8). Gemeinsam mit dem gleichlautenden Vollverb geht das ,light verb` auf einen stark unterspezifizierten Lexikoneintrag zurück. Dieser enthält keine Argumentstruktur, sondern umfasst lediglich eine nicht strikt festgelegte Ansammlung elementarer semantischer Informationen, wie der Eintrag zu engl. give zeigt:

181 Kövecses (2000: 94) sieht in Fügungen wie Angst haben ebenfalls ein metaphorisches Übertragungsmuster am Werk, nämlich ATTRIBUTES ARE POSSESSED OBJECTS. Was unter „Possession“ zu verstehen ist, d. h. ob damit lediglich die Besitzrelation gemeint ist oder ob Possession noch andere Relationstypen umfasst, wird aber nicht ausreichend deutlich gemacht. 
Give [...] agentive, some entity (concrete or abstract) is to be transferred to a recipient/goal.

Dieser Eintrag zu give kann zusätzlich durch Weltwissen angereichert werden, im Falle von give etwa durch die Information, dass ein ,geben'-Ereignis (im Regelfall, aber nicht notwendigerweise) jemandem zugute kommt (vgl. Butt 2010: 73f.). Da ein unterspezifizierter Verbeintrag wie der von give sowohl als ,light verb“ als auch als Vollverb realisiert sein kann, spricht Butt (2010: 74) auch von einem „verbal passe-partout“, s. auch Abb. 4. Sie geht davon aus, dass es in den Sprachen der Welt „somewhere between five and twenty“ dieser Passepartouts gibt (2010: 72).

Unterspezifizierter Passepartout

- mit elementaren semantischen Informationen

- mit Fragmenten von Weltwissen

- ohne Argumentstruktur

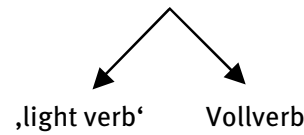

Abb. 4: Unterspezifizierter Lexikoneintrag für FV und Vollverb (vgl. Butt/Lahiri 2013: 24)

Wird dieser verbale Passepartout als Vollverb gebraucht, entfaltet er eine Argumentstruktur auf der Basis der in ihm enthaltenen semantischen Informationen. Wird er hingegen als ,light verb“ verwendet, muss sein semantischer Gehalt mit der Hauptprädikation im Satz verbunden werden: ,[...] argument merger [...] must take place, as well as a modulation of the main event semantics by the information coming from the light verb" (2010: 74).

Die Annahme eines einzigen Lexikoneintrags, auf dem sowohl Vollverb als auch, light verb/FV basieren, ist aufgrund seiner Beschreibungsökonomie zwar grundsätzlich attraktiv. Allerdings ist Butts These mit einer problematischen Stipulation verbunden: In gängigen Modellen der lexikalischen Semantik werden Verbbedeutungen (und Bedeutungen von Substantiven) generell mittels unterspezifizierter Lexikoneinträge beschrieben (vgl. Bierwisch 1983: 85f.; Jackendoff 1983: 95-106 und 1990: 34; Pustejovsky 1995: 62). Postuliert man nun für ein relativ kleines Set von Verben eine exklusiv gültige Art der Unterspezifikation, hätte man in einem übergreifenden Modell des Lexikons zwei völlig divergierende Formen unterspezifizierter Lexikoneinträge vorzusehen, nämlich zum einen Einträge, welche die möglichst lesartenübergreifend gültigen elementaren 
Bedeutungskomponenten einschließlich einer Argumentstruktur enthalten, sowie zum anderen Einträge, die Butt (2010: 73f.) zufolge lediglich eine lose Ansammlung unterschiedlicher Informationen darstellen und die keine Argumentstruktur besitzen, dafür aber mit Fragmenten von Weltwissen angereichert sind. Die Annahme zweier gänzlich unterschiedlicher Formen von Lexikoneinträgen erscheint indes kaum sinnvoll.

Der Vorschlag von Butt ist auch deshalb kritisch zu sehen, weil der Weg, der von dem Passepartout zum ,light verb“ führen soll, alles andere als klar ist. Wenn das Verb als ,light verb“ in die Syntax eingeführt wird, findet, so Butt (2010: 74), „argument merger“, d. h. eine Verschmelzung der Argumente von Hauptprädikation und Passepartout, statt. Dabei erschließt sich indes nicht, wie dann gleichzeitig behauptet werden kann, dass die Passepartouts angeblich gar keine Argumentstruktur aufweisen (,the content of this entry will be not an argument structure“ [Butt 2010: 73]). Argumentstrukturen können naheliegenderweise nur dann miteinander verschmelzen, wenn auch tatsächlich zwei solcher Strukturen vorhanden sind.

Butt (2010: 59-64) und Butt/Lahiri (2013: 9-11) versuchen auch den syntaktischen Status von ,light verbs` $\mathrm{zu}$ bestimmen. Sie gehen davon aus, dass es sich weder um ein Vollverb noch um ein Auxiliar, sondern um eine distinkte syntaktische Kategorie handelt (Butt/Lahiri 2013: 14). Als Argument hierfür werden vor allem die jeweils eigenen distributionellen Eigenschaften des ,light verbs`angeführt, die es sowohl vom Vollverb als auch vom Auxiliar abgrenzen. Dies wird bei Butt (2010: 63f.) u. a. an Beispielen aus dem Urdu und dem Mandarin exemplifiziert, es ist im Hinblick auf die Abgrenzung zwischen FV und Vollverb aber auch gut auf die Befunde aus dem Deutschen anwendbar. So wurde in Kapitel 5.2 bereits auf die besonderen syntaktischen Beschränkungen hingewiesen, denen $\mathrm{z}$. B. bringen als FV gegenüber dem Vollverb unterliegt (mangelnde Erfragbarkeit, fehlende Möglichkeit der pronominalen Wiederaufnahme usw.). Von der Klasse der Hilfsverben sind ,light verbs'/FV laut Butt/Lahiri (2013: 11) u. a. aufgrund flexivischer Eigenschaften abzugrenzen: Auxiliare könnten in defektiven Paradigmen auftreten, bei ,light verbs‘ sei das nie der Fall. Zudem seien ,light verbs‘ auch im Hinblick auf ihre Funktion deutlich von Auxiliaren unterschieden:

The functional work done by auxiliaries is to situate the main event predication with respect to temporal or aspectual information, that is, with respect to speech and reference time [...]. This is not the functional work carried by light verbs, which modify the main predication in some (usually subtle) manner, but do not situate it with respect to speech and reference times.

(Butt/Lahiri 2013: 23) 
Als weiteres Argument gegen eine Einordnung der ,light verbs‘ als Auxiliare wird angeführt, dass diese im Gegensatz zu den Hilfsverben historisch persistent seien; Auxiliare seien dagegen überwiegend das Ergebnis von Grammatikalisierungsprozessen: „[...] light verbs generally resist language change, while auxiliaries are amenable to them“ (Butt/Lahiri 2013: 26).

Ob die These von Butt/Lahiri (2013), dass ,light verbs‘ sich von Voll- und Hilfsverben grundsätzlich abheben und eine eigene syntaktische Kategorie bilden, auf die FV des Deutschen zutrifft, ist nicht leicht zu beurteilen. FV sind in dieser Arbeit anders definiert, und auch die in Kapitel 2 vorgestellten Definitionsversuche der germanistischen Forschung sind nur teilweise vergleichbar. Als ein Unterschied gegenüber der traditionellen Forschung kann aber festgehalten werden, dass Butt/Lahiri (2013) die Aktionsartenmarkierung offenbar nicht als Funktion der ,light verbs‘ beschreiben. Dies steht in deutlichem Widerspruch zu der herkömmlichen Sicht auf diese Verbklasse, in der gerade die Aktionsartenmarkierung als zentrale Eigenschaft von FV herausgestellt wurde (Genaueres dazu in Kapitel 2.2.1). Damit wird freilich die in dieser Untersuchung vertretene Ansicht gestützt, der zufolge die Aktionsartenmarkierung eine eigenständige grammatische Funktion darstellt, die mit dem Bereich der FVG allenfalls am Rande etwas zu tun hat.

Eine eingehende Überprüfung der von Butt/Lahiri (2013) aufgestellten Hypothese, dass ,light verbs‘ historisch stabil seien, würde sich auch für die FV nach der hier vertretenen Definition lohnen. Auf den ersten Blick erscheint es jedoch unwahrscheinlich, dass, light verbs ‘ FV generell eine Insel für Sprachwandelprozesse bilden, wie Butt/Lahiri (2013: 26) annehmen. Zwar mag es zutreffen, dass Auxiliare überwiegend durch Grammatikalisierungsprozesse entstehen, während es ,light verbs'/FV in gewisser Weise schon immer gegeben hat (vgl. zum Althochdeutschen den Überblick bei Schrodt 2004: 71f.). Letztere sind jedoch ebenfalls semantischem Wandel unterworfen, wie ein Blick in die historischen Wörterbücher unmissverständlich klar macht (zu bringen s. ${ }^{2} \mathrm{DWB} \mathrm{Bd}$. 5, Lfg. 5 , 817-830, zu finden ${ }^{2}$ DWB Bd. 9, 505; zum umfangreichen und sich historisch nach und nach entfaltenden Verwendungsspektrum von gehen s. ${ }^{1} \mathrm{DWB} \mathrm{Bd}$. 4,1,2, 2376-2475). Wenn Butt/Lahiri (2013) betonen, dass ,light verbs“ nicht in Grammatikalisierungsprozessen auftreten, hat dies nicht notwendigerweise etwas mit ihrer Resilienz gegen sprachlichen Wandel zu tun. Es bedeutet nur, dass sie einer bestimmten Art von Sprachwandel, der Grammatikalisierung, nicht in dem Maße unterliegen wie z. B. Auxiliare. Da gar nicht klar ist, inwieweit ,light verbs‘/FV überhaupt eine Komponente der Grammatik darstellen, ist es wenig überraschend, dass sie nur schwer als Resultat von Grammatikalisierungsprozessen beschrieben werden können. Für lexikalischen Wandel sind ,light verbs‘/FV aber - 
nach allem, was man bisher weiß - nicht weniger empfänglich als andere Verben.

\subsubsection{FV im Konstruktionsnetzwerk}

Auf der Grundlage der oben erörterten Forschungspositionen hat sich bisher keine eindeutige Antwort auf die Frage ergeben, ob für FV ein eigener Lexikoneintrag anzusetzen ist oder ob ein gemeinsamer Eintrag für FV und Vollverb angesetzt werden kann. Im Folgenden sollen Argumente dafür vorgebracht werden, dass ein einziger Lexikoneintrag genügt. Diese Position ist vor allem deshalb bevorzugt als These zu überprüfen, weil sie die ökonomischste Repräsentation darstellt. Als Beschreibungsrahmen für die Validierung der These sei die Konstruktionsgrammatik gewählt. Dies ist deshalb sinnvoll, weil sich der Konstruktionsbegriff, wie in Kapitel 4.3 gezeigt, grundsätzlich als geeignet für die Modellierung von FVG erwiesen hat. Ein konstruktionsgrammatischer Ansatz ist für die hier im Mittelpunkt stehende Frage aber auch insofern von Vorteil, als die Konstruktionsgrammatik von der Arbeitshypothese ausgeht, dass das Inventar der sprachlichen Einheiten - seien es Wörter oder eben Konstruktionen - keine Liste, sondern ein strukturiertes Gebilde darstellt, das als Netzwerk modelliert werden kann. Wenn es um eine Positionsbestimmung der FV bzw. FVG im Sprachsystem geht, lassen sich im Kontext eines Netzwerkmodells somit wesentlich interessantere Hypothesen formulieren, als wenn man lediglich von einer listenartigen Repräsentation ausgeht.

\subsubsection{Konstruktionen im Netzwerk: theoretische Vorüberlegungen}

Eine der Grundannahmen der Konstruktionsgrammatik besteht darin, dass die Konstruktionen ein Netzwerk bilden (vgl. Goldberg 1995: 67; Jackendoff 2002: 184f.; 2008: 15f. sowie den Forschungsüberblick in Fischer/Stefanowitsch 2006: 11). Konstitutiv für das Netzwerk ist ein hierarchischer Aufbau: Es gibt Typen und Untertypen von Konstruktionen (Mutter- und Tochterknoten), und beide sind durch top-down verlaufende Vererbungsrelationen miteinander verbunden. Daher gilt: „Die Gesamtheit der grammatischen Eigenschaften der Einzelkonstruktionen ist [...] die Summe von ererbten allgemeinen und konstruktionsspezifischen idiosynkratischen Faktoren“ (Webelhuth 2011: 158).

Während die Modellierung des Konstruktionsinventars als Netzwerk mehr oder weniger Gemeingut der unterschiedlichen konstruktionsgrammatischen Theorieversionen darstellt (vgl. Engelberg/Holler/Proost 2011: 12), weichen die verschiedenen Ausprägungen der Konstruktionsgrammatik hinsichtlich der 
Frage, wie die Vererbungsmechanismen beschaffen sind, voneinander ab. Kay (2000: 20) geht davon aus, dass die Eigenschaften eines Mutterknotens vollständig und ausnahmslos an den Tochterknoten weitergegeben werden (daher spricht man hier auch von „complete mode of inheritance“, vgl. Goldberg 1995: 73). Wenn in der Vererbungshierarchie Konflikte zwischen untergeordneten und übergeordneten Informationen auftreten, führe dies, so die Annahme Kays, zu nicht wohlgeformten Strukturen. Im Gegensatz dazu legen Goldberg (1995: 73f.) und Croft (2001: 25-28) ihrem Konstruktionsnetzwerk den sog. ,normal mode of inheritance" zugrunde, der aus der Forschung zur Künstlichen Intelligenz stammt (auch als Default-Vererbung bezeichnet, vgl. St. Müller 2010: 400). In diesem Modell ist auch partielle Vererbung gestattet. Als Beispiel für diese Art der Vererbung kann die Konzepthierarchie Vogel herangezogen werden. Hier besteht sowohl zwischen den Konzepten VOGEL $\rightarrow$ SPATZ als auch zwischen den Konzepten VoGEL $\rightarrow$ PINGUIN eine Vererbungsrelation. Da aber im Fall VogEL $\rightarrow$ PINGUIN der Tochterknoten PINGUIN Spezifikationen aufweist, die mit zentralen Eigenschaften des übergeordneten Knotens VoGeL im Konflikt stehen, werden nur solche Merkmale weitervererbt, die mit den Merkmalen des Tochterknotens übereinstimmen (HAT EINEN SCHNABEL, Hat FlÜGEL, nicht jedoch KANN FLIEGEN). Im normalen Vererbungsmodus wird Information somit solange an untergeordnete Knoten weitergegeben, bis eine Eigenschaft des untergeordneten Knotens die Vererbung unterbindet. Für die hier zu leistende Positionsbestimmung von FV und FVG sei der partielle Vererbungsmodus zugrunde gelegt, da er als psychologisch plausibel gelten kann (vgl. Croft/Cruse 2004: 275f.).

Das konstruktionelle Netzwerkmodell, mit dem hier gearbeitet werden soll, erlaubt grundsätzlich auch „multiple inheritance“ (Goldberg 1995: 73) bzw. „multiple parents“ (Croft 2001: 25f.). Eine Konstruktion kann somit von mehreren übergeordneten Konstruktionen erben. Solche Mehrfachvererbungen werden von Croft (2001: 26) am Beispiel des Satzes I didn't sleep illustriert, vgl. Abb. 5. ${ }^{182}$

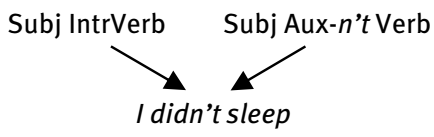

Abb. 5: Mehrfachvererbung („multiple inheritance“)

182 Wie Croft (2001: 26) allerdings selbst bemerkt, ist I didn't sleep keine Konstruktion, sondern eine konkrete Äußerung, d. h. ein „construct“ im Sinne von Kay (2002: 4). 
Für die Modellierung von Mehrfachvererbung ist allerdings ein weiterer Relationstyp vorzusehen, der über die bloße Vererbungsbeziehung, wie sie in Abb. 5 dargestellt ist, hinausgeht. So stellt z. B. die Verknüpfung von Verben mit den von Goldberg (1995: 3-5) postulierten abstrakten Argumentstrukturkonstruktionen (Transitiv, Ditransitiv usw.) eine Fall von „multiple inheritance“ dar. Goldberg (1995: 50) behandelt solche Verknüpfungen von Verben mit schematischen Konstruktionen unter dem Terminus „fusion“. So erbt das Konstrukt to mail someone something sowohl von der Ditransitivkonstruktion [[SUBJ V OBJ1 OBJ2]/[X CAUSES Y TO RECEIVE Z] ] ${ }^{183}$ als auch von dem Verb mail, für welches Goldberg einen verbspezifischen Frame <mailer mailed mailee> annimmt. Im Zuge dieser Verknüpfung werden die Rollen < mailer mailed mailee $>$ mit dem abstrakten Rollengerüst der Ditransitivkonstruktion identifiziert und fusioniert. Dass mail allerdings überhaupt mit der Ditransitivkonstruktion verschmolzen werden kann, ist darauf zurückzuführen, dass mail und der Ditransitiv in einer besonderen semantischen Relation stehen; diese wird bei Goldberg (1995: 50f., 59-65) als „R-Relation“ bezeichnet. Da die Ditransitivkonstruktion eine Bedeutung trägt - in diesem Fall die schematische Transferbedeutung [X CAUSES Y TO RECEIVE Z] (Goldberg 1995: 53) -, kann mail als eine spezifische Ausprägung des allgemeinen Transferschemas aufgefasst werden. Beide Ausgangskonstruktionen stehen somit in einer taxonomischen oder „instance“-Relation (Goldberg ebd.), vgl. Abb. 6.

$\mathrm{R}$

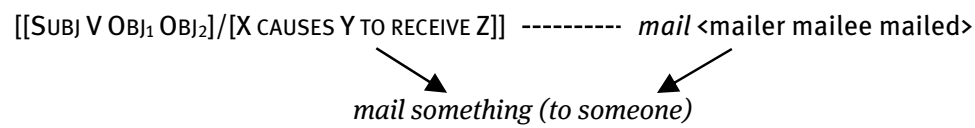

R: Instanz (,to mail ist eine spezifische Art des Transfers')

Abb. 6: R-Relation ,Instanz'

Die Rolle der R-Relation lässt sich somit in der Weise beschreiben, dass diese selbst keine Vererbungsrelation darstellt, sondern lediglich plausibel macht, weshalb genau diese beiden Knoten und nicht beliebige andere in einer Vererbungsbeziehung zu einem gemeinsamen Tochterknoten stehen. Für R-Links

183 Die Konstruktion ist hier nach dem in Croft (2001) verwendeten Schema [[SYNTAX]/[SEMANTIK]] notiert; damit ist die Konstruktion als Zeichen gefasst, das eine syntaktische Spezifikation mit Inhalt verbindet, s. auch die Skizze der Konstruktionsgrammatik in Kapitel 4.3. 
nimmt Goldberg verschiedene Subtypen an: Instanz-, Teil-Ganzes- und metaphorische Relationen (vgl. Goldberg 1995: 74-81). Das Konstruktionsnetzwerk wird somit durch zwei Link-Typen konstituiert: Vererbungslinks und R-Links. ${ }^{184}$

Die Annahme von Mehrfachvererbung bringt allerdings mit sich, dass auch Konflikte zwischen vererbenden Elternknoten auftreten können. Goldberg (1995: 97f.) nennt als Beispiel eine bestimmte Untergruppe der Resultativa des Englischen, hier (8), die variable Wortstellung erlaubt, obwohl dies bei Resultativa eigentlich nicht der Fall ist, wie (9b) vs. (9a) zeigt.

a. He cut short the speech.

b. He cut the speech short.
a. She hammered the metal flat.
b. *She hammered flat the metal.

(Goldberg 1995: 97)

Das für Resultativkonstruktionen besondere Verhalten in (8) sieht Goldberg als Fall von Mehrfachvererbung: Die Konstruktion in (8) erbt sowohl von der Resultativkonstruktion als auch von der Partikelverbkonstruktion, die etwa in (10) vorliegt.

(10) a. Break the cask open.

b. Break open the cask.

(Goldberg ebd.)

Da die Resultativkonstruktion für die AP grundsätzlich keine Wortstellungsvariation AP erlaubt, die Partikelverbkonstruktion aber sehr wohl, liegt ein Konflikt zwischen beiden Elternknoten vor. Dass hinsichtlich der Wortstellung die eine Elternkonstruktion gewinnt, während die andere unterliegt, bedarf somit einer Erklärung. Goldberg sieht allerdings keine Möglichkeit einer prinzipiengesteuerten Lösung dieses Vererbungskonflikts. Sie nimmt Konflikte dieser Art vielmehr zum Anlass, eine besondere Variante des Netzwerkmodells zu propagieren, nämlich ein sog. „full-entry“-Modell. In einem solchen Modell werden Vererbungen nicht als online-Prozesse aufgefasst, sondern als statische Korrespondenzrelati-

184 Hierzu lassen sich die beiden Relatortypen „Beziehungsindikator“ und „Prozeßindikator“ anführen, die in der von M. Job entwickelten netzwerksemantischen Beschreibungssprache für semantischen Wandel (vgl. Job/Job 1997) eine entscheidende Rolle spielen. 
onen zwischen vollspezifizierten Einträgen. Dass die Verben in (8) sich hinsichtlich ihrer Wortstellungseigenschaften an die variable Partikelverbkonstruktion und nicht an die eigentlich strenger restringierte Resultativkonstruktion anlehnt, ist im Tochterknoten selbst spezifiziert:

[T] he information about the specific construction is provided in the specific construction, even if it is redundant with the information contained in (one of) the parent constructions.

(Croft/Cruse 2004: 277)

Da der Tochterknoten damit letztlich autark ist, sind Vererbungskonflikte zwischen Elternknoten für diesen irrelevant.

Theoretisch und praktisch wirft das full-entry-Modell allerdings einige Fragen auf, auf die zumindest kurz hinzuweisen ist: Wenn Croft/Cruse (2004: 276) im Anschluss an Goldberg (1995) die Notwendigkeit eines full-entry-Modells damit zu begründen versuchen, dass Konflikte zwischen Elternknoten nicht nach bestimmten Regeln lösbar seien (,there is no principled way to choose which parent would win in a conflict“, ebd.), kann dies ein solches Modell kaum ausreichend rechtfertigen. Die Tatsache, dass wir im Rahmen eines klassischen vererbungsbasierten Netzwerkmodells etwa bei den resultativen Partikelverbkonstruktionen in (8) nicht genau nachvollziehen können, weshalb der Konflikt genau in der vorliegenden Weise gelöst wird, heißt noch nicht, dass das entsprechende Netzwerkmodell dadurch falsifiziert und stattdessen ein anderes, in diesem Fall ein Modell mit voll spezifizierten Knoten, zu präferieren wäre. Möglicherweise sind bei diesem und auch bei anderen Vererbungskonflikten die jeweils zugrundeliegenden Lösungsprinzipien schlicht noch unentdeckt. Die Ablehnung des klassischen Vererbungsmodells durch Goldberg (1995) und Croft/Cruse (2004) basiert somit auf einem unzulässigen argumentum ad ignorantiam.

Ein weiteres Problem des full-entry-Modells liegt darin, dass es lediglich feststellt, dass sprachliche Strukturen so sind, wie sie sind. Wenn es sich bei Vererbungsrelationen um bloße Korrespondenzen handelt, die nichts mehr erklären müssen, verliert das Netzwerkmodell erheblich an theoretischer Attraktivität. Anstelle eines Netzwerks mit Vererbungsrelationen würde auch eine Liste von voll spezifizierten Einzeleinträgen genügen. Die auch von Goldberg (1995) und Croft/Cruse (2004) vertretene Grundannahme der Konstruktionsgrammatik, der zufolge die Gesamtheit aller Konstruktionen ein strukturiertes Inventar bildet, würde damit im Grunde überflüssig: Ein listenartiges, unstrukturiertes Inventar von ,full entrys' wäre vollkommen ausreichend. 
Als Fazit dieser Gegenüberstellung von Vererbungsmodellen lässt sich festhalten, dass bei der Beschreibung der Vererbungsstrukturen von FVG von den folgenden netzwerktheoretischen Grundannahmen auszugehen ist:

- Es wird der „normal mode of inheritance“ oder sog. Default-Vererbung vorausgesetzt; das full-entry-Modell wird nicht verwendet, da es keine überprüfbaren Vorhersagen leistet.

- Dem hier präferierten Modell entsprechend wird vollständige Vererbung aller übergeordneten Informationen an die untergeordneten Knoten als Normalfall postuliert. Die Möglichkeit einer Mehrfachvererbung zweier gleichgeordneter Elternknoten an eine Tochter ist ebenfalls vorgesehen.

- Tritt ein Konflikt zwischen Eltern- und Tochterknoten auf, kann vom Normalfall der vollständigen Vererbung abgewichen werden, indem partielle Vererbung stattfindet oder - anders formuliert - die Vererbung derjenigen Informationssegmente blockiert wird, die mit den entsprechenden Komponenten des Tochterknotens nicht kompatibel sind.

- Zwischen Elternknoten wird ein besonderer Relationstyp - eine sog. „R-Relation“ - vorausgesetzt (z. B. Typ-Instanz- oder Teil-Ganzes-Relation); die RRelation legt fest, welche Elternknoten gemeinsam an eine Tochter vererben.

\subsubsection{Vererbungsstrukturen bei FVG}

Wenn man Konstruktionen als konventionalisierte Verbindungen von phonologischer, syntaktischer und semantischer Information begreift (vgl. Kapitel 4.3.2), so lässt sich ein konkretes FVG wie einen Kuss geben als Kombination dreier Informationsschichten Phonologie, Syntax und Semantik notieren (vgl. Jackendoff 1997: 49; 2008: 26; Kay 2002: 4).

(11) Phon jmdm. einen Kuss geben

Syn [vP V NP NP]

Sem ENERGIETRANSFER <AG, PAT, REZ>

Für die hier relevanten Zwecke ist es ausreichend, wenn die Werte der einzelnen Positionen in (11) nur angedeutet werden: Die phonologische Komponente wird informell durch eine Wörterbuchnennform, die syntaktische durch eine elementare Strukturübersicht und die semantische Position verkürzt durch ein Prädikat ENERGIETRANSFER indiziert. Mit dieser Angabe des Sem-Werts wird ausgedrückt, dass jmdm. einen Kuss geben analog zum einfachen Verb küssen semantisch als „verb of impact by contact“ klassifiziert werden kann, dem eine Grundstruktur CAUSE (x, BECOME (BE AT (y, z))) entspricht (dazu ausführlich Kapitel 5.3.3.1). Bei 
diesem Transferereignis erhält ein Rezipient (das Dativobjekt) von einem Agens ausgehende KÜSSEN-Energie (diese ist das Transferierte, d. h. der Patiens).

In vergleichbarer Weise wird auch die NP einen Kuss notiert (diese wäre ihrerseits auf zwei Elternknoten Artikel + N zurückzuführen, was hier der Einfachheit halber unberücksichtigt bleibt), wobei für Kuss als Nominalisierung ebenfalls ein Ereigniskonzept angesetzt wird.

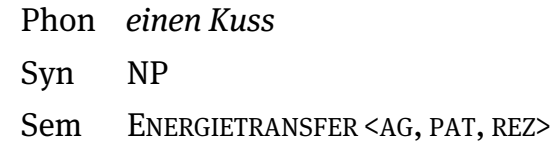

Einträge wie in (11) und (12) werden in der Konstruktionsgrammatik nicht nur für Phrasen, sondern auch für Einzelwörter angenommen, da Wörter prinzipiell denselben Status wie syntaktische Konstruktionen besitzen: In beiden Fällen liegt ein Sprachzeichen mit einer phonologischen und semantischen Schicht sowie einer syntaktischen Spezifikation vor - „it's constructions all the way down“, wie Goldberg (2006: 18) für das gesamte Kontinuum zwischen Lexikon und Syntax postuliert (vgl. auch Goldberg 2006: 5 sowie Jackendoff 1997: 49). Wörter sind daher ebenfalls nach dem genannten Muster zu notieren, vgl. den Eintrag in (13) für geben, hier mit einer verkürzten Sem-Angabe TRANSFER (zu einer detaillierten Beschreibung der Bedeutung von geben s. Kapitel 5.3) und mit einer Spezifikation der syntaktischen Kategorie als V sowie der Argumentstruktur als $<\mathrm{NP}_{\text {Sbj }} \mathrm{NP}_{\text {obj1 }}$ $\mathrm{NP}_{\text {Obj2 }}>$. ${ }^{185}$

$$
\begin{aligned}
& \text { (13) Phon geben } \\
& \text { Syn } \mathrm{V}<\mathrm{NP}_{\text {Sbj }} \mathrm{NP}_{\mathrm{Obj} 1} \mathrm{NP}_{\mathrm{Obj} 2}> \\
& \text { Sem TRANSFER <AG, PAT, REZ> }
\end{aligned}
$$

In einem nach den oben beschriebenen Prinzipien aufgebauten Netzwerk sind die Konstruktionen (11), (12) und (13) so miteinander in Beziehung zu setzen, dass das FVG einen Kuss geben sowohl von geben als auch von einen Kuss erbt (,multiple inheritance“). Zusätzlich zu den beiden Vererbungssträngen ist noch eine

\footnotetext{
185 Zur Notation: Argumentstrukturen, d. h. phonologisch nicht spezifizierte Kombinationen, werden in spitze Klammern < > eingeschlossen; dies gilt sowohl für syntaktische wie für semantische Argumentstrukturen (dazu Goldberg/Jackendoff 2004: 564). Reale syntaktische Einheiten werden mit [ ] notiert.
} 
R-Relation anzusetzen, welche die Elternknoten miteinander verbindet. In diesem Fall liegt eine taxonomische Similaritätsrelation ${ }^{186}$ zwischen dem allgemeinen TRANSFER-Konzept, das geben ausdrückt, und dem spezifischen ENERGIETRANSFER vor, der im Kuss-Ereignis gegeben ist (vgl. auch Kapitel 5.3.1). Die über eine R-Relation verbundenen Sem-Stränge können deshalb auch wie in (14) beschrieben werden.

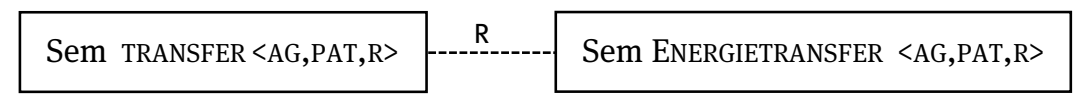

Wichtig für das Folgende ist allerdings, dass die Informationsschichten Phon, Syn und Sem, die hier per Konvention ein sprachliches Zeichen bilden, prinzipiell eigenständig sind und auch eigenständig in die Vererbungsstruktur eintreten (vgl. das Konzept der „parallelen Architektur“ bei Jackendoff 1997: 39; 2002: 198). Daher sind potentiell drei Vererbungsstränge von den Eltern- zu den Tochterknoten vorzusehen.

Die Darstellung in (15) modelliert die Position des FVG jmdm. einen Kuss geben im Netzwerk.

(15)

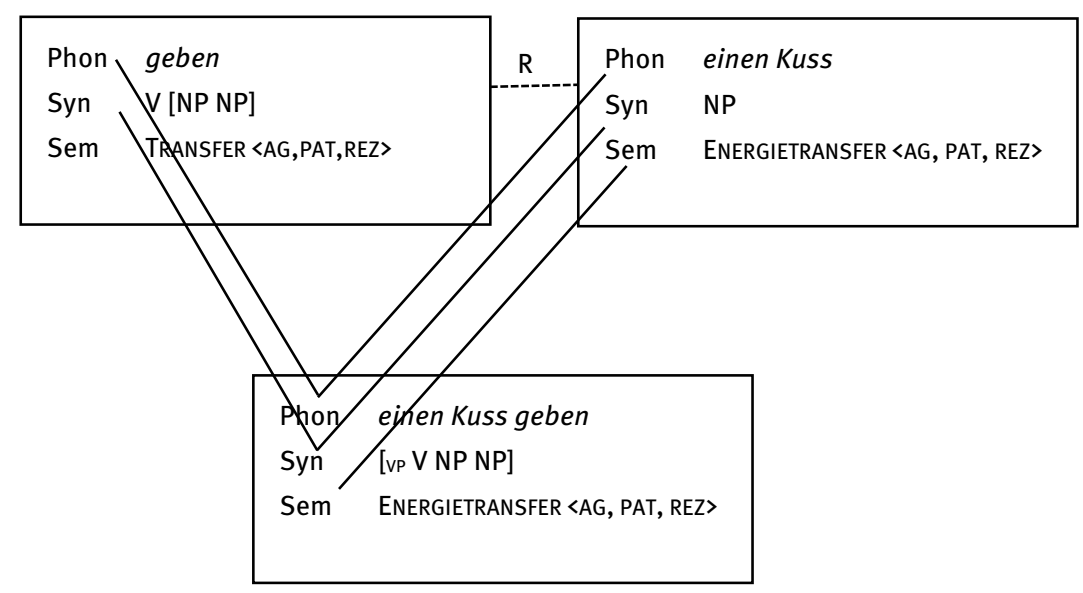

$\mathrm{R}=$ taxonomische Relation

$186 \mathrm{Zu}$ taxonomischen Relationen als Form der Similaritätsrelation vgl. Harm (2015: 71) mit weiterer Literatur. 
Freilich fehlt hier noch so etwas wie eine Phrasenstrukturregel, die Verb und NP zu einer Phrase verbindet, oder - je nach theoretischem Standpunkt - eine Unifizierung bzw. eine „Merge“-Operation (zu diesen Alternativen St. Müller 2010: 86f., 141-143). Auf diesen Punkt kommt es hier indes nicht an, da in unserem Zusammenhang vor allem die Sem-Werte von Interesse sind. Hier ist an der Modellierung in (15) nämlich entscheidend, dass der Sem-Wert von geben nicht vererbt wird; dafür wird die Bedeutung der NP einen Kuss an das FVG weitergegeben, und zwar einschließlich der Quantifikation des Ereignisses. Die Vererbung des Nominalstranges verläuft somit ungehindert, während die Vererbung des Verbstranges blockiert ist. In Bezug auf die Vererbung der Rollengerüste lässt die Modellierung in (15) zwei Optionen zu, da die Rollen <AGENS, PATIENS, REZIPIENT> im Prinzip sowohl vom Verbstrang als auch vom Nominalstrang vererbt werden können. Wesentliche Unterschiede in der Modellierung ergeben sich aus diesen beiden Optionen allerdings kaum.

Im Rahmen eines full-entry-Modells scheint diese Vererbungsblockade auf den ersten Blick kein Problem darzustellen: In der Tochterkonstruktion ist spezifiziert, dass sie die semantische Information des Verbs nicht erbt, sondern nur die formalen Eigenschaften von bringen übernimmt. Wie oben angedeutet, ist diese Lösung allerdings theoretisch wenig befriedigend, da sie den Befund lediglich beschreibt, nicht aber erklärt. Sie ist auch deshalb problematisch, weil das full-entry-Modell nicht vorhersagen kann, weshalb bei einer großen Zahl von FVG nur eine bestimmte Gruppe von Verben - nämlich stark schematische Verben wie geben, haben, bringen und machen - den Mutterknoten bilden. Wenn im Tochterknoten eines vollspezifizierten Eintrags festgeschrieben ist, dass die semantischen Informationen des Verbs nicht übernommen werden, ist es unerheblich, welches Verb der übergeordnete Knoten enthält. Eine Netzwerkdarstellung mit voll spezifizierten Einträgen und einem Default, Verbbedeutung wird bei FVG nicht vererbt‘ kann die Generalisierung, dass FVG vorzugsweise mit bestimmten Verben auftreten, somit nicht erfassen - es ist hier schlicht egal, um welches Verb es sich handelt.

Innerhalb einer netzwerktheoretischen Darstellung, die sich nicht mit einer bloßen Konstatierung des Vorhandenen zufrieden gibt, wäre also erstens zu erklären, weshalb hier überhaupt ein Vererbungskonflikt vorliegt, und zweitens wäre darzulegen, weshalb dieser Konflikt in genau der Weise gelöst wird, dass der Verbknoten partiell blockiert, der Nominalknoten jedoch vollständig weitergegeben wird.

Zum ersten Punkt: Der Konflikt, der hier vorliegt, ist in der R-Relation begründet. Da Verb und Nomen in einer Typ-Instanz-Relation stehen, enthalten sie redundante Information: Durch das Verb geben wird ein allgemeines Konzept 
,Transfer‘ ausgedrückt, und das Nomen Kuss enthält eine spezifische Ausprägung dieses schematischen Konzepts, nämlich ,Energietransfer mit körperlichem Kontakt‘ (s. Kapitel 5.3.2). Die Information, die das Verb enthält, stellt somit eine Teilmenge der Information dar, die das Nomen als der merkmalhaltigere Knoten bietet. Diese Redundanz führt bei gleichzeitiger Vererbung an einen gemeinsamen Tochterknoten zu einer nicht wohlgeformten Äußerung - es läge gewissermaßen der Transfer eines Transfers vor. Die beiden Elternknoten befinden sich also gerade deshalb miteinander im Konflikt, weil sie tautologisch sind.

Die Tatsache, dass zur Lösung dieses Konflikts die Vererbung des verbalen und nicht des nominalen Sem-Werts blockiert wird, steht nun durchaus im Einklang mit der Standardannahme, dass bei einer Vererbung alle Informationen von der übergeordneten Ebene an die untergeordnete weitergegeben werden. Der Grund dafür ist in dem eben skizzierten Verhältnis der beiden Sem-Werte zu sehen: Da die Bedeutung des nominalen Knotens alle semantischen Informationen enthält, die das Verb ebenfalls aufweist, und es gleichzeitig semantisch reichhaltiger ist, genügt es, wenn allein der nominale Knoten vererbt wird. Das Verb weist demgegenüber keine semantische Information auf, über die das Nomen nicht auch verfügte. Durch die Blockierung des verbalen Knotens entsteht somit auch kein Informationsverlust im Netzwerk, und eine Verletzung der Standardannahme „Alles wird vererbt“ wird dadurch vermieden, dass der semantische Gehalt des Verbs quasi stellvertretend durch das Nomen weitergegeben wird. Eine Konfliktlösung, die den umgekehrten Weg einschlüge und dem Verb gegenüber dem Nomen den Vorzug in der Vererbung gäbe, würde den Default „Alles wird vererbt“ hingegen klar verletzen, da mit einer Blockierung des nominalen Konzepts auch alle weiteren Informationen, die dieses zusätzlich zu denen des Verbs enthält, verloren gingen. ${ }^{187}$

In Bezug auf die hier angenommene Vererbungsblockade ist allerdings noch einmal in aller Deutlichkeit darauf hinzuweisen, dass der verbale Knoten nicht in Gänze blockiert wird, sondern lediglich die Weitergabe der semantischen Schicht unterbunden wird; der Phon- und der Syn-Wert werden uneingeschränkt an die Tochter vererbt. Da für diese Werte keine Redundanzen bestehen und folglich auch kein Konflikt vorliegt, erfolgt die Vererbung ungehindert.

Dass Blockaden des Bedeutungsstrangs bei ungestörter Weitergabe der anderen Informationspositionen durchaus geläufig sind, zeigt im Übrigen auch ein

187 Die hier geschilderte Blockierung der Informationsweitergabe im Netzwerk weist Parallelen zur sog. „Elsewhere condition“ (Kiparsky 1973: 94) aus der Phonologie und Morphologie auf, der zufolge eine spezifische Regel die Anwendung einer allgemeineren Regel blockiert. 
Blick auf Phraseologismen. So können Verbindungen wie einen Narren an jemandem/etwas fressen, jemanden sehr gern haben' oder jmdm. ein Schnippchen schlagen ,jmds. Absichten durchkreuzen' netzwerktheoretisch ebenfalls dahingehend interpretiert werden, dass die Verben fressen bzw. schlagen zwar alle grammatischen Eigenschaften der freien Verben erben, die Vererbung der semantischen Merkmale aber unterbunden bzw. stark eingeschränkt ist.

An diesem Punkt kann eine Zwischenbilanz gezogen werden: Eine netzwerktheoretische Beschreibung, wie sie hier für das Beispiel jmdm. einen Kuss geben vorgestellt wurde, scheint zumindest prinzipiell in der Lage zu sein, das oben angesprochene Paradoxon eines semantisch leeren Verbs, das trotz seiner semantischen Leere Selektionseigenschaften besitzt, aufzulösen. Dies ist möglich, wenn man beide Eigenschaften - die Selektion der Nomina durch das Verb und dessen Bedeutungsleere - auf unterschiedlichen Hierarchieebenen des Netzwerks verortet. Die semantische Selektion ist dabei auf der Ebene der Elternknoten anzusiedeln. Hier stehen Einträge mit vollständig spezifizierten Bedeutungspositionen einander gegenüber; dass diese Selektionsrelationen untereinander aufweisen können, ist erwartbar. Die Bedeutungsleere des Verbs im FVG ist in diesem Fall lediglich das Resultat einer Vererbungsblockade, die sich im Tochterknoten und somit eine Hierarchieebene tiefer manifestiert. In einem hierarchischen Netzwerk ist der Widerspruch eines bedeutungsleeren Verbs, das nach semantischen Gesichtspunkten selektiert, somit gar nicht erst existent.

Daraus folgt auch, dass für das FV und das entsprechende Vollverb ein einziger Lexikoneintrag ausreichend ist. Vererbt das Verb geben gemeinsam etwa mit einer NP ein Buch an einen Tochterknoten ein Buch geben, so liegt keine Similarität zwischen den Sem-Werten und folglich auch keine Redundanz vor: $G e$ ben drückt ein Ereigniskonzept Transfer aus, während die NP ein Buch ein Gegenstandskonzept kodiert, das als transferiertes Objekt auf das Verbalereignis bezogen werden kann. Die R-Relation ist somit nicht als Similarität, sondern als Kontiguität zwischen den involvierten Konzepten $\mathrm{zu}$ bestimmen. ${ }^{188} \mathrm{Im}$ Fall einer Kontiguitätsrelation besteht, netzwerksemantisch betrachtet, jedenfalls keine Redundanz zwischen den Informationen der beiden Knoten. Diese vererben ihre semantischen Informationen daher jeweils vollständig und uneingeschränkt an die Tochter-Phrase jmdm. ein Buch geben.

Mit dem FVG jmdm. einen Kuss geben wurde ein Beispiel mit tautologischem Bedeutungsaufbau gewählt (vgl. die Klassifikation in Kapitel 3). Die FVG mit konversem Bedeutungsaufbau, d. h. Fügungen des Typs zur Aufführung bringen, zei-

188 Zum Gegensatz Similarität/Kontiguität vgl. Raible (1981/2011: 24f.). 
gen hinsichtlich der hier wirksamen Vererbungsmechanismen jedoch partiell abweichende Eigenschaften. Zwar besteht auch hier eine semantische Similarität zwischen dem FV und dem Nomen; das Nomen Aufführung ist jedoch passivisch - die Aufführung von Hamlet ist das ,Aufgeführtwerden von Hamlet', s. dazu Kapitel 3.2.4 -, das Verb bringen und das gesamte FVG sind demgegenüber aktivisch. Der verbale Mutterknoten hat mit der Bedeutungskomponente ,aktivisch also sehr wohl eine wichtige Information an die Tochter weitergegeben. Auch dies ist vor dem Hintergrund des hier zugrunde gelegten Netzwerkmodells vollkommen erwartbar: Das Prinzip „Alles wird vererbt“ wird sehr strikt eingehalten. Dies führt eine Dekomposition der betreffenden Sem-Werte vor Augen:" ${ }^{189}$


b. bringen: [X CAUSE (BECOME Y $(\mathrm{BE}(\mathrm{z})))$ ]

Von den semantischen Informationen des Verbs in (16b) wird genau das Prädikat weitervererbt, das nicht auch im Nomen Aufführung enthalten ist, nämlich „X CAUSE“, das BECOME-Prädikat hingegen ist doppelt vorhanden, weshalb die Blockade greift. Ein eigener Lexikoneintrag für das FV bringen ist folglich auch hier nicht nötig, FV und Vollverb bringen sind vielmehr als identisch anzusehen.

\subsection{Zur Modellierung der FVG}

Während die Frage nach der Repräsentation der FV mit einem vergleichsweise hohen argumentativen Aufwand zu beantworten war, scheint es für FVG zunächst vollkommen unstrittig, dass jeweils eigene Lexikoneinträge vorzusehen sind. Dies belegen FVG wie eine Entscheidung fällen oder eine Frage stellen, die eine einmalige Kombination von Verb und Nomen aufweisen bzw. nur in wenigen vergleichbaren Fügungen vorhanden sind. Auch für FVG wie zur Aufführung bringen, Antwort geben oder eine Reise machen kann man durchaus annehmen, dass diese einzeln gespeichert sind. Dies liefe jedoch auf das Postulat einer bloßen Liste von FVG hinaus - FVG wären damit wie Phraseologismen repräsentiert. Eine solche Liste ist freilich theoretisch wenig befriedigend: Das Ziel einer Wissenschaft ist, Muster zu ermitteln oder Regeln zu formulieren, die so allgemein

189 Um die Parallelität zwischen den Resultatszuständen besser sichtbar zu machen, orientiert sich die Beschreibung an Jackendoff (1990), der BE AT für unterschiedliche semantische Felder verwendet, s. o. Kapitel 5.5.5.2. 
sind, wie die Daten es zulassen. Dass eine sprachliche Einheit gespeichert werden kann, ist zudem kein Argument gegen die Existenz einer Regel, die diese Einheit hervorgebracht hat.

Ein Argument dafür, dass FVG nicht einfach wie geläufige Phraseologismen repräsentiert sind, ergibt sich schon aus der syntaktischen Struktur der Bildungen. FVG nutzen aus dem großen Inventar möglicher Satzmuster nur eine relativ kleine Anzahl von Grundstrukturen: Transitiv, Ditransitiv und Lokalsatz. Bei Phraseologismen ist dagegen keine derart deutlich erkennbare Beschränkung auf bestimmte syntaktische Muster erkennbar. Hier herrscht große Vielfalt, wie die Beispiele in (17) illustrieren:
a. wissen, wo Barthel den Most holt (Satzgefüge)
b. ins Gras beißen (Lokalsatz)
c. jmdm. ein X für ein U vormachen (Ditransitiv + PP)
d. bedient sein (Kopulasatz)
e. eine ruhige Kugel schieben (Transitiv mit obligatorischem Attribut zur Objekt- NP)
f. die Kartoffeln aus dem Feuer holen (Transitiv + lokale Angabe)

Eine weitere Eigenheit der FVG gegenüber Phraseologismen mit verbalem Kopf besteht darin, dass bei Letzteren keine Präferenzen bei der Auswahl der Verben erkennbar sind, wie die Heterogenität der Verben in (17) sowie die Beispiele in (18) zeigen.

(18) Maulaffen feilhalten, am Rad drehen, jmdm. auf den Magen schlagen, in der Patsche stecken, jmdn. über den Löffel balbieren

FVG dagegen werden überwiegend mit Verben wie kommen, gehen, bringen, machen oder geben konstruiert. Dabei handelt es sich, wie Winhart (2005: 113) betont hat, um Verben, die kein manner-Argument enthalten (vgl. gehen im Gegensatz $\mathrm{zu}$ schleichen, s. o. 8.1.1). Zudem kodieren diese Verben elementare Konzepte („humanly relevant scenes“ Goldberg 1995: 39-43; vgl. 2006: 78). Die genannten Verben kommen zwar auch in Phraseologismen vor (dem Affen Zucker geben, keinen Aufstand machen usw.); sie sind hier jedoch nicht in der Weise vorherrschend wie bei den FVG. Ein starkes Argument dafür, dass FVG mit der Annahme einer Liste von Einzelrepräsentationen nicht ausreichend beschrieben sind, liefern nicht zuletzt die in Kapitel 5 herausgearbeiteten Beschränkungen bei der Kombination von Verb und Nomen. Diese lassen in der Tat eine Regel vermuten, die hinter den Einzelvorkommen steht. 
Wenn somit einerseits für eine große Gruppe von FVG als wahrscheinlich gelten muss, dass eine entsprechende Regel existiert, bleibt es andererseits dabei, dass für FVG wie eine Entscheidung fällen oder eine Frage stellen ein individueller Eintrag anzunehmen ist. Es gibt somit offenbar beides nebeneinander: regelhafte Muster, welche Einzelvorkommen generieren, sowie Einzeleinträge, die nicht als Output einer generellen Regel beschrieben werden können.

Die paradoxe Situation, dass die Repräsentation eines syntaktischen Musters sowohl idiomatische Einzelansätze als auch Regeln erfordert, könnte zunächst dahingehend aufgelöst werden, dass man sich für eine dieser Repräsentationsformen - Einzeleintrag oder Regel - entscheidet, die dann als grundlegend zu gelten hätte. Der erstgenannte Weg wird in stark „gebrauchsbasierten“ Ausprägungen der Konstruktionsgrammatik beschritten (zu dieser Theorievariante Engelberg/Holler/Proost 2011: 13). Hier werden lediglich die abgespeicherten Einzelvorkommen als zentral für das sprachliche Wissen betrachtet, während Generalisierungen oder Regeln als sekundär oder sogar als Ergebnis von HokusPokus-Linguistik erachtet wird: „Linguists cannot second-guess the sort of generalizations speakers make beyond these constructions [d. h. Generalisierungen jenseits einzelner Verbkonstruktionen, etwa im Hinblick auf allgemeinere Argumentstrukturmuster, V. H.]“ (Croft 2003: 64; vgl. auch Boas 2008: 137f.). Im Rahmen solcher Ansätze werden allenfalls Generalisierungen auf verhältnismäßig niedrigem Niveau postuliert, etwa in Form von „Idiomverbänden“ (Zeschel 2008: 263f.), die einzelne Vorkommen nach Ähnlichkeit zu kleineren Clustern gruppieren. ${ }^{190}$

Auf der anderen Seite des sprachwissenschaftlichen Theoriespektrums steht die Regelkomponente im Mittelpunkt der Hypothesenbildung. Als rezente Ansätze sind vor allem das „Minimalistische Programm“ im Anschluss an Chomsky (1995) sowie die sog. „Distributed Morphology“ (begründet von Halle/Marantz 1993) zu nennen. Das zuletzt genannte Paradigma verfährt in Bezug auf das Lexikon besonders radikal: Dieses ist letztlich nur als Liste von Morphemen (bzw. „Wurzeln“ bei Marantz 1997: 203f.) konzipiert, und es werden keine lexikalischen Regeln angenommen, da die Regelkomponente vollständig mit der Syntax zusammenfällt. Das Lexikon ist hier lediglich ein „appendix to the grammar, a list of basic irregularities“ im Sinne von Bloomfield (1933: 274). Phraseologismen, die in konstruktionsbasierten Modellen gleichberechtigt neben den Regeln stehen, sind sowohl im Minimalismus als auch in der Distributed Morphology sekundär.

190 Zeschel (2008) legt dies anhand von FVG des Deutschen dar. Da dieser Untersuchung aber keine klare Definition von FVG zugrunde gelegt ist, verwundert es nicht, dass keine weitgehenden Generalisierungen postuliert werden. 
Für diese Beschreibungsansätze besteht dann allerdings die Notwendigkeit, auch opake Phraseologismen wie den Musterfall engl. kick the bucket kompositional zu analysieren (zu Einzelheiten G. Müller 2011: 213f.; vgl. auch Marantz 1997: 212; McGinnis 2002: 669). Dass dies nur mit sehr starken und psychologisch wenig plausiblen Zusatzannahmen möglich ist - etwa einzelnen Lexikoneinträgen für phraseologisches kick, the und bucket -, liegt auf der Hand.

Einen dritten Weg zwischen den hier skizzierten Theorietraditionen, die jeweils entweder die Regel oder das Einzelexemplar absolut setzen, hat Jackendoff (2008) aufgezeigt, und zwar am Beispiel der Nominalreduplikation des Englischen (z. B. in day by day oder book after book), die er als „family of constructions“ analysiert (zum Konzept der „family of constructions“ auch Goldberg/Jackendoff 2004). Bei der Nominalreduplikation - Jackendoff (2008: 8) bezeichnet sie als „NPN-construction“ - sind insofern ganz ähnliche Verhältnisse wie bei den FVG gegeben, als auch hier idiomatische Einzelvorkommen (z. B. eye to eye) neben produktiven Mustern, sog. ,pockets of productivity“ (Jackendoff 2008: 18), stehen (hier ist die produktive Konstruktion $\mathrm{N}$ after $\mathrm{N}$ zu nennen, die z. B. in student after student vorliegt). Das Konzept der Konstruktionsfamilie bietet sich somit als geeigneter Ansatzpunkt für die Beschreibung von FVG an. (Eine auszugweise Darstellung der Konstruktionsfamilie NPN des Englischen in Form einer Vererbungshierarchie bietet Abb. 7.)

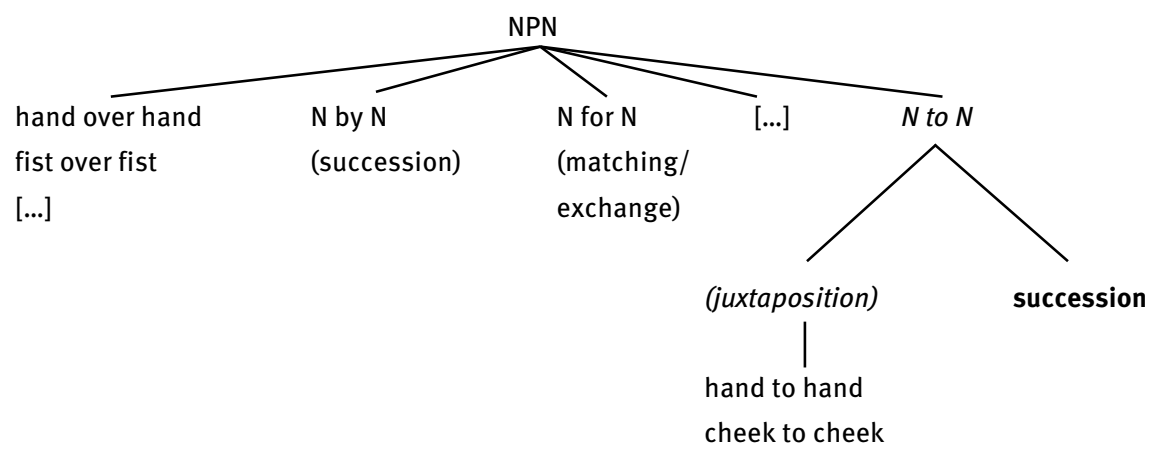

fett: produktives Muster

kursiv: semiproduktives Muster

nicht-fett: Idiom

Abb. 7: Die Konstruktionsfamilie engl. NPN (Auszug aus Jackendoff 2008: 17) 
Wie bereits angedeutet, zeichnet sich dieses Modell wesentlich dadurch aus, dass es keine strikte Trennung zwischen Speicher und Regel vornimmt: Regelhaft erzeugte Strukturen, vor allem wenn häufig auf sie rekurriert wird, können auch in der Speicherkomponente enthalten sein und müssen nicht stets aufs Neue online erzeugt werden. Regeln machen exemplarbezogenes sprachliches Wissen also keinesfalls überflüssig, sondern Exemplar und Regel können nebeneinander bestehen (vgl. Jackendoff 2002: 159f.; Goldberg/Jackendoff 2004: 535; Goldberg 2006: 12). Nicht zuletzt sind auch die Regeln selbst in irgendeiner Weise im Gedächtnis gespeichert. Regelbasiertes und exemplarbasiertes sprachliches Wissen ist daher prinzipiell von derselben Art, und beides kann auf dieselbe Weise beschrieben werden, nämlich als konventionalisierte Verbindung semantischer, syntaktischer und phonologischer Information. Der Eintrag für eine idiomatische NPN-Verbindung unterscheidet sich folglich in der Grundstruktur nicht von dem Eintrag für eine regelhaftes „constructional idiom“, wie z. B. für den Subtyp N after $\mathrm{N}$ in (19).

$\begin{array}{ll}\text { Meaning } & \text { MANY } \mathrm{X}_{j} \mathrm{~S} \text { IN SUCCESSION [...] } \\ \text { Syntax } & {\left[\mathrm{N}_{\mathrm{i}} \mathrm{P}_{\mathrm{j}} \mathrm{N}_{\mathrm{i}}\right]} \\ \text { Phonology } & \mathrm{Wd}_{\mathrm{i}} \text { after }_{\mathrm{j}} \mathrm{Wd}_{\mathrm{i}}\end{array}$

(Jackendoff 2008: 26)

Von einem singulär repräsentierten Phraseologismus weicht (19) lediglich darin ab, dass in der phonologischen Schicht der Stellvertreter „Wd“ (d. h. „Word“) statt einer konkreten Realisierung eingesetzt ist. Das Lexikon ist in diesem Modell folglich alles andere als ein ,appendix of the grammar“ im Sinne Bloomfields; es enthält vielmehr Wissenskomponenten, die traditionellerweise eher der Grammatik zugeschrieben werden:

[...] it [das Lexikon, V.H.] is a repository of whatever pieces of linguistic structure have to be stored in long term memory. Some of these pieces are relatively idiosyncratic, and some include variables that must be filled productively or semiproductively in the course of building a sentence in working memory [...]. From this perspective [...], there is no distinction between in the grammar and in the lexicon, there are only distinctions of productivity in the lexicon.

(Jackendoff 2008: 18) 
Inwieweit eine sprachliche Erscheinung regulär ist, bemisst sich, wie das Zitat deutlich macht, allein nach deren Produktivität. Jackendoff (2008) unterscheidet dabei zwischen drei Produktivitätsstufen:

- unproduktive/idiomatische Bildungen

- semiproduktive Muster

- produktive Muster

Idiomatische Verbindungen sind naturgemäß singulär (vgl. das erwähnte Beispiel eye to eye, das vorwiegend in der Verbindung mit see vorkommt; s. Jackendoff 2008: 11). Als produktiv beschreibt Jackendoff dagegen Fügungen mit den Präpositionen after, for und upon (vgl. term after term, dollar for dollar, book upon book). Diese lassen jedes Nomen in der N-Position zu, sofern die grundlegenden Beschränkungen (kein Massenomen, keine Determinierer) nicht verletzt sind. Als semiproduktiv werden hingegen Konstruktionen aufgefasst, deren akzeptable Repräsentanten gelernt werden müssen. Kennzeichnend für semiproduktive Muster ist dementsprechend, dass neue Repräsentanten auch als neuartig erkannt werden (,one recognizes novel cases, such as foot by foot, as relatively unusual“ [2008: 16]; zu der Unterscheidung zwischen produktivem und semiproduktivem Muster s. auch Jackendoff 2002: 155-167).

Versucht man nun für die FVG des Deutschen unterschiedliche Produktivitätsstufen im Sinne dieses Modells herauszuarbeiten und mit Einträgen wie in (20) zu beschreiben, so ist für ein singulär vorkommendes FVG wie ein Urteil fällen die folgende Basisstruktur anzunehmen:

\begin{tabular}{|c|c|c|}
\hline$(20)$ & Sem & URTEILEN $_{\mathrm{I}}\left(\right.$ Agens $_{\mathrm{j}}$, Patiens $\mathrm{k}$ ) \\
\hline & Prag & Figur $_{\mathrm{i}}$ vs. Grund $\mathrm{m}_{\mathrm{m}}$ \\
\hline & Syn & $\mathrm{NP}_{\mathrm{j}} \mathrm{V}_{\mathrm{m}} \mathrm{NP}_{\mathrm{i}}\left(\mathrm{PP}_{\mathrm{k}}\right)$ \\
\hline & Phon & $\mathrm{Wd}_{\mathrm{j}}$ fällen $_{\mathrm{m}}$ ein Urteil $\mathrm{i}_{\mathrm{i}}\left(\ddot{\text { uber }} \mathrm{Wd}_{\mathrm{k}}\right)$ \\
\hline
\end{tabular}

Durch den Index „¡“ “ wird hier deutlich gemacht, dass der Sem-Wert nicht nur für das gesamte FVG ein Urteil fällen gilt, sondern auch für die NP Urteil - diese doppelte Relation zwischen Ausdruck und Inhalt ist schließlich das kennzeichnende Merkmal von FVG (vgl. Kapitel 3.2.1; zur Position „Prag(matik)“ Welke 2019: 53).

Wenn in einem nächsten Schritt semiproduktive FVG zu ermitteln und als Lexikoneinträge darzustellen sind, stellt sich allerdings die Frage, wie „Semiproduktivität“ dingfest gemacht werden kann, noch einmal mit besonderer Dringlichkeit. Jackendoffs oben angeführte Beschreibung, der zufolge semiproduktive Regelhaftigkeit daran zu erkennen sei, dass das jeweilige Exemplar als neuartig 
empfunden werde, erscheint impressionistisch, da es sich auf subjektive Kompetenzurteile einzelner Sprecher verlässt. Man kann sich aber darauf verständigen, dass Fügungen, die in der Position des nominalen Arguments anstelle eines einzelnen phonologisch spezifizierten Nomens eine Liste von Kandidaten enthalten, hinsichtlich ihrer Produktivität irgendwo zwischen Einzelidiom und unbeschränkt produktivem Muster stehen (zum Zusammenhang zwischen Liste und Semiproduktivität s. auch Jackendoff 2002: 161).

Aus dem Bereich der FVG wäre als Kandidat für ein semiproduktives Muster die Konstruktion mit zollen zu nennen. Eine Stichprobe der ersten hundert Treffer in DEREKo (07.01.2016) ergibt hier interessante Häufigkeitsverteilungen: Am häufigsten ist Respekt mit 41 Treffern, gefolgt von Anerkennung mit 16 und Lob mit 14 Treffern. Daneben gibt es eine Handvoll singulär bezeugter Nomina: Beifall (1), Hochachtung (2), Ovationen (1), Standing Ovations (1), Dankeschön (1), Applaus (1), Interesse (1). Der Rest der zollen-Fügungen (22) entfällt auf Tribut zollen. Dieses spielt insofern eine Sonderrolle, als es polysem ist: Zum einen bedeutet es ,schuldige Hochachtung erbringen', zum anderen wird es in der Bedeutung ,einem negativen Umstand Rechnung tragen' verwendet (Stark verbessert zeigte sich Lorraine Lißmann, die aber den wuchtigen Aufschlägen ihrer Gegnerin Tribut zollen musste Braunschweiger Zeitung, 14.02.2013). Die FVG mit zollen gruppieren sich somit um einen Kernbestand von drei sehr häufig auftretenden Lexemen (Respekt, Anerkennung, Lob). In einem Lexikoneintrag für das zollen-FVG ist dies als Liste mit den betreffenden Nomina zu repräsentieren, die aber prinzipiell offen ist, vgl. (21).

$$
\begin{array}{ll}
\text { Sem } & \text { EINE POSITIVE EINSTELLUNG ÄUßERN }_{\mathrm{I}}\left(\text { Agens }_{\mathrm{j}}, \text { Rezipient }_{\mathrm{k}}\right) \\
\text { Syn } & \mathrm{NP}_{\mathrm{j}} \mathrm{V} \mathrm{NP}_{\mathrm{k}} \mathrm{NP}_{\mathrm{i}} \\
\text { Phon } & \mathrm{Wd}_{\mathrm{i}}\{\text { Respekt, Anerkennung, Lob, ... }\} \text { zollen }
\end{array}
$$

Produktiv ist dieses FVG in der Weise, dass im Einzelfall Synonyme oder Quasisynonyme zu den Lexemen der Liste gebildet werden. Produktivität ist hier exemplarbasiert: Das Nomen Respekt oder Anerkennung wird im Einzelfall durch Hochachtung o. ä. ersetzt.

Für die in Kapitel 5 behandelten FVG mit bringen, geben, haben und machen sind kaum solche Listen wie in (21) anzunehmen. Die Zahl der in Betracht kommenden Nomina ist hier zu groß für eine Repräsentation des Phon-Wertes als Lexemliste. Freilich stellt sich die Frage, ob sie denn auch groß genug ist für die Annahme eines phonologisch nicht-spezifizierten Nominalslots und damit auch eines voll produktiven Musters. Mit einer uneingeschränkt produktiven Konstruktion wie z. B. der enumerativen Verbindung dt. $\mathrm{N}$ für $\mathrm{N}$ ist das bringen-FVG 
nicht vergleichbar: Im Fall der N für N-Konstruktion scheinen der Besetzbarkeit keine Grenzen gesetzt (Neologismus für Neologismus, Salzstreuer für Salzstreuer usw.), sofern die Beschränkung, dass kein Massenomen insertiert werden darf ('Mehl für Mehl), nicht verletzt ist. Beim bringen-FVG sind die Grenzen der Besetzbarkeit hingegen relativ eng gezogen, wie die Ausführungen in Kapitel 5 gezeigt haben. Dies ist in diesem Fall allerdings noch kein Argument für eine listenartige Repräsentation der Nomina. Zwar erscheint das bringen-FVG im Vergleich zur genannten N für N-Konstruktion wenig produktiv. Dennoch ist es nicht wesentlich anders als solche stark produktiven Konstruktionen zu repräsentieren.

Zur Begründung der Hypothese, dass das bringen-FVG trotz der Beschränkungen in der Leerstellenbesetzung voll produktiv ist, seien einige grundsätzliche Überlegungen zu Konstruktionen angeführt. Mit Kay (2002: 1) kann man Konstruktionen generell dadurch kennzeichnen, dass sie nicht blind gegenüber den Wörtern sind, die in die Leerstellen der Konstruktion eintreten können; dies hebt Konstruktionen zugleich von Regeln ab, welche in der Tat blind sind für das lexikalische Material, das sie verarbeiten (so kann z. B. jedes Nomen unabhängig von seiner Bedeutung Subjekt des Satzes sein oder durch einen Relativsatz ergänzt werden). Die Beschränkungen in der Füllung der Leerstellen ergeben sich direkt aus dem Zeichencharakter von Konstruktionen: Wenn eine syntaktische Konfiguration mit einem bestimmten Inhalt verbunden ist, dürfen die Leerstellen nicht mit Wörtern besetzt sein, welche mit der Konstruktionsbedeutung inkompatibel sind. ${ }^{191}$

Dies lässt sich an einem einfachen Beispiel illustrieren: Die Konstruktion V, bis der Arzt kommt (Richter/Sailer 2009) bezeichnet eine exzessiv ausgeübte Tätigkeit. Für die Besetzung der V-Position kommen hier nur solche Verben in Betracht, die hinsichtlich der Intensität, mit der die Handlung ausgeführt wird, gradierbar sind. Die Beispiele in (22) sind daher völlig unproblematisch, während die Verben in (23) sich mit der Konstruktionsbedeutung ,etwas exzessiv ausüben ‘ nicht vereinbaren lassen bzw. die Kombination eine humoristische Wirkung hat, wie in (23a) der Fall.

191 In der Frage, ob Konstruktionen eine Bedeutungsseite aufweisen müssen, gehen die Auffassungen auseinander. Langacker (1987) und Goldberg (1995) nehmen prinzipiell an, dass Konstruktionen eine Inhaltsseite haben, während Jackendoff sich in diesem Punkt eher zurückhaltend zeigt: „My own preference [...] is to think that not all syntactic configurations are inherently meaningful“ (2002: 179; vgl. 2008: 25). Auch Kay (2002: 1f.) beschreibt das Vorhandensein von semantischer Information nicht als notwendiges Merkmal. Dennoch setzen sowohl Jackendoff (2008) als auch Kay (2002) für die meisten Konstruktionen, die sie beschreiben, eine Bedeutungskomponente an, auch wenn diese zum Teil vage bleibt. 
(22) Wir feiern/tanzen/trinken/schreiben/kicken bis der Arzt kommt.

(23) a. 'Wir promovieren, bis der Arzt kommt.

b. *Wir kommen an, bis der Arzt kommt.

c. \#Wir verteilen den Kuchen, bis der Arzt kommt.

Dass die Konstruktionsbedeutung der Leerstellenbesetzung Restriktionen auferlegt, wird in konstruktionsgrammatischen Beschreibungen nicht immer gebührend berücksichtigt. So besteht für die NPN-Konstruktion sowohl des Englischen als auch des Deutschen u. a. die Einschränkung, dass die Nomina nicht determiniert sein dürfen (Jackendoff 2008: 9). Dies wird bei Jackendoff (ebd.) als idiosynkratische Eigenschaft der Konstruktion, als konstruktionsspezifische Abweichung von der Standardsyntax stipuliert. Eine solche Stipulation ist freilich möglicherweise gar nicht nötig, sofern man das Fehlen eines Determinierers von der Bedeutung der Konstruktion her motiviert. Wenn die $\mathrm{N}$ für N-Konstruktion eine All-Quantifikation ausdrückt (Tag für Tag fuhr sie zur Arbeit, jeden Tag in unendlicher Folge'), so wäre determiniertes *einen Tag für einen Tag oder *den Tag für den Tag jedenfalls wesentlich schwerer mit der Bedeutung, unendliche Sukzession` zu vereinbaren, da Determinierer stets spezielle Annahmen über Definitheit oder Bekanntheit im Diskursuniversum transportieren (s. auch Kapitel 6.3.2). Für den Ausdruck unendlicher Sukzession sind diese Annahmen aber eher störend. Ohne dass hier weiter auf Einzelheiten der NPN-Konstruktion eingegangen werden kann, dürfte deutlich geworden sein, dass grammatische Eigenschaften nicht voreilig als irregulär und damit konstruktionsspezifisch postuliert werden sollten. Die Regularitätsannahme sollte auch für Konstruktionen als Nullhypothese gelten. Es ist daher stets zu prüfen, ob sich die grammatischen Eigenschaften einer Konstruktion nicht von deren Bedeutung her begründen lassen, bevor diese als formale Idiosynkrasien im Lexikoneintrag festgeschrieben werden.

Bei der Besetzung der N-Position in FVG gibt es relativ starke Beschränkungen der geschilderten Art: Der implikative Bedeutungsaufbau bzw. die FigurGrund-Struktur kommt nur dadurch zustande, dass das Ereignis als Nomen kodiert wird, zu welchem ein inhaltsleeres Verb einen maximalen Kontrast bildet (s. Kapitel 7.3.2). Als inhaltsleer kann das Verb aber nur dann ausgewiesen werden, wenn eine Similaritätsrelation zwischen Nomen und Verb besteht und das Verb zugleich weniger spezifisch als das Ereignisnomen ist. Nur wenn diese Bedingungen gegeben sind, kann die Vererbung des verbalen Sem-Strangs blockiert werden, so dass der einschlägige Bedeutungsaufbau und der damit verbundene pragmatische Effekt zustande kommt. Dreh- und Angelpunkt des Aufbaus von FVG ist also die Beschränkung der Nomina auf solche, die mit dem 
Verb in einer Similaritätsrelation stehen. Dieser Forderung ist umso schwerer zu genügen, je größer die semantische Komplexität des Verbs ist. In der Tat besteht offenbar eine Korrelation zwischen der Komplexität der Verbbedeutung und der Zahl der in die N-Position einsetzbaren Nominalisierungen. So ist geben semantisch reichhaltiger als haben, wie bereits die Glossierung von geben als „x causes y to have z“ zeigt, die einfaches „have“ als Teilbedeutung enthält (s. dazu Kapitel 5.3.2). Dementsprechend gibt es zumindest in den hier zugrunde gelegten Daten offensichtlich deutlich mehr FVG mit haben (vor allem im Bereich der Einstellungsausdrücke des Typs eine Meinung haben) als mit geben. Die Verbindungen mit machen, das semantisch schlicht als Do-Prädikat zu beschreiben ist, sind wesentlich häufiger als Verbindungen mit bringen, welches mit der Prädikatskonstellation CAUSE \& BECOME (BE (AT)) eine vergleichsweise merkmalhaltige Semantik aufweist. Gleichwohl ist zu berücksichtigen, dass die Produktivität eines FVG auch von anderen Faktoren abhängt. Hier ist besonders die Forderung zu nennen, dass FVG auch syntaktisch wohlgeformte Äußerungen ergeben müssen (vgl. den in Kapitel 5.3.3 beschriebenen Akzeptabilitätskontrast zwischen seine $\mathrm{Zu}$ stimmung geben und *seine Befürwortung geben). Neben den Input-Constraints, die regeln, welches Nomen aufgrund seiner Bedeutung in die Fügung eintreten kann, bestehen somit auch Output-Constraints. Beide Arten von Beschränkungen beeinflussen die Produktivität des jeweiligen Bildungsmusters.

Wenn das Zustandekommen des implikativen Bedeutungsaufbaus bzw. des Figur-Grund-Kontrastes als der wesentlichen funktionalen Eigenschaft von FVG daran geknüpft ist, dass nur bestimmte Nomina in die Konstruktion eintreten, stellt sich die Frage, ob diese Beschränkungen im Lexikoneintrag für das FVG an irgendeiner Stelle explizit zu machen sind. Dies ist jedoch nicht erforderlich: Der Figur-Grund-Kontrast bzw. die implikative Bedeutungsstruktur sind notwendige Eigenschaften der Konstruktion, auf die hin alle anderen Eigenschaften der Konstruktion ausgerichtet sind - so auch die Leerstellenbesetzung. Die Semantik bzw. Pragmatik diktiert quasi automatisch die Selektion. Diese muss daher auch nicht expliziert werden. Gleiches gilt übrigens auch für die oben als Beispiel angeführte Verbindung V, bis der Arzt kommt. Dass hier nur Verben vorkommen können, deren Semantik in irgendeiner Weise eine Intensivierung der Handlungsausübung enthält, ergibt sich unmittelbar aus der Konstruktionsbedeutung und muss nicht eigens festgeschrieben werden. Die Tatsache, dass das hier als Beispiel ausgewählte bringen-FVG alles in allem weniger produktiv erscheint als die NPN-Konstruktion, ist somit auf die verhältnismäßig starken Restriktionen zurückzuführen, welche die Konstruktionsbedeutung der Besetzung der N-Position auferlegt. Unbeschadet der vergleichsweise geringen Zahl von Leerstellen- 
füllern ist das bringen-FVG durchaus ein produktives Muster im Sinne Jackendoffs, da es für die Leerstellungbesetzung keine Liste von Kandidaten benötigt wie das in (20) dargestellte semiproduktive Muster zollen $+\mathrm{N}$.

Von hier aus zurückblickend stellt sich aber auch für die zollen-FVG die Frage, ob in diesem Fall wirklich eine Liste angenommen werden muss. Die Konstruktionsbedeutung, eine positive Einstellung äußern'schreibt genau vor, welche Nomina in Frage kommen. Aus diesem Grund könnte man auf die Angabe einer Liste verzichten. Dies hätte allerdings den Nachteil, dass dann alle Kandidaten - ob Beifall, Dankeschön oder Lob - gleichberechtigt wären. Gerade dies ist jedoch nicht der Fall. Das deutliche Ungleichgewicht in der Produktivität kann eine Liste mit den häufigsten Besetzungen der Nominalposition letzten Endes am besten erklären.

Wenn hier für die bringen-FVG sowie für die Fügungen mit haben, geben und machen keine Liste postuliert wird, schließt dies freilich nicht aus, dass es auch in diesem Bereich exemplarbasierte Produktivität geben kann, wie sie primär für die semiproduktiven Bildungen vorauszusetzen ist - Regel und Speicherung können, wie oben gezeigt, koexistieren. So ist z. B. denkbar, dass die Fügung zur Uraufführung bringen eine Variante des häufigen zur Aufführung bringen darstellt. Da Uraufführung aber genauso wie alle anderen Nomina des Paradigmas die semantischen Beschränkungen erfüllt, die das Verb erfordert, kann es ebenso gut regelhaft generiert sein. Gerade die bringen-FVG, deren Nomina aus vielen semantisch sehr heterogenen Wortfeldern stammen (vgl. Druck, Aufführung, Versteigerung, Anwendung, Verrechnung, Ausstrahlung), zeigen aber sehr deutlich, dass dieses Muster kaum auf einer von Exemplar zu Exemplar fortschreitenden Erweiterung beruht. Für diese Bildung hat man also nicht nur aufgrund der relativ hohen Zahl der Kandidaten, sondern auch wegen deren semantischer Heterogenität ein produktives Konstruktionsmuster anzunehmen.

Im Hinblick auf die Frage, wie ein Lexikoneintrag für ein FVG mit bringen, haben, geben oder machen $\mathrm{zu}$ formulieren ist, wurde hier zunächst die phonologische Spezifikation thematisiert. Neben der Phon-Position wirft aber auch die Sem-Position Fragen auf: Während bei einem semiproduktiven FVG eine konkrete lexikalische Bedeutung angegeben werden kann - im Fall von zollen + N so etwas wie ,eine positive Einstellung äußern` -, ist dies bei den produktiven FVG nicht möglich. Dies zeigt sich wiederum am deutlichsten bei den bringen-Fügungen, bei denen kaum ein sinnvoller Oberbegriff etwa sowohl zu Druck als auch zu Versteigerung gefunden werden kann. Was ist also als Wert der Sem-Position anzugeben, wenn kein konkretes Konzept benannt werden kann? Mehr als eine allgemeine Charakterisierung als „Ereigniskonzept“ ist nicht möglich: 
(24) Sem Ereigniskonzept (Agens $_{\mathrm{i}}$, Patiens $\left.\mathrm{s}_{\mathrm{k}}\right)$

Prag Figur vs. Grund $_{m}$

Syn $\quad \mathrm{NP}_{\mathrm{j}} \mathrm{V}_{\mathrm{m}} \mathrm{NP}_{\mathrm{k}} \mathrm{PP}_{\mathrm{i}}$

Phon $\mathrm{Wd}_{\mathrm{j}}$ bringen $_{\mathrm{m}} \mathrm{Wd}_{\mathrm{k}}$ zur/zum $\mathrm{Wd}_{\mathrm{i}}$

Die entscheidenden semantischen Relationen sind hier jedoch in den Indizes ausgedrückt: Das Verb, bezeichnet durch „m“, hat keine Entsprechung im SemStrang, es ist lediglich in der Phonologie sowie in der Syntax spezifiziert; es ist auch für die Pragmatik der Fügung relevant, da die fehlende lexikalische Bedeutung des Verbs den Figur-Grund-Effekt erst auslöst. Das Zustandekommen dieses Effekts ist somit auf ein komplexes Bedingungsgefüge zurückzuführen, das bei der Similarität zwischen Verb und Nomen seinen Ausgang nimmt:

Similarität Nominalkonzept/Verbalkonzept

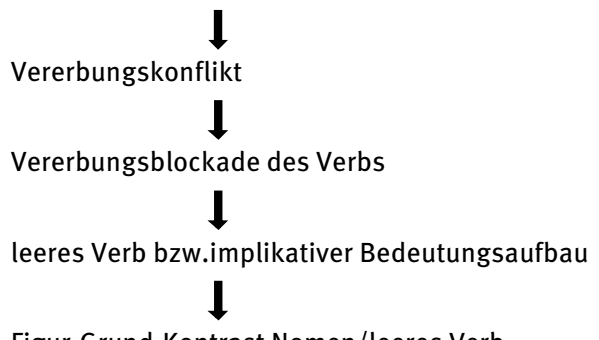

Figur-Grund-Kontrast Nomen/leeres Verb

Abb. 8: Voraussetzungen für den Figur-Grund-Effekt

Die von Jackendoff (2008) untersuchte Konstruktionsfamilie NPN, aber auch die Konstruktionsfamilie „Resultativum“ (Goldberg/Jackendoff 2004) weisen gewissermaßen als ,Oberhaupt‘ ein phonologisch vollständig offenes Muster auf, $d . h$. eben $\mathrm{N}_{\mathrm{i}} \mathrm{PN}_{\mathrm{i}}$ für die Nominalreduplikation und [NP V NP XP] für die Resultativkonstruktion. Im letztgenannten Fall steht XP entweder für eine AP (etwa in Willy watered the plants flat) oder für eine PP (The critics laughed the play off the stage; vgl. Goldberg/Jackendoff 2004: 536). Wenn hier die FVG des Deutschen ebenfalls als Konstruktionsfamilie beschrieben werden, stellt sich die Frage, ob es auch für FVG eine phonologisch nicht spezifizierte Repräsentation dieser Art gibt, der sich dann alle weiteren Fälle als Instanzen zuordnen ließen. Der Annahme einer schematischen ,Überkonstruktion' stehen zunächst einige Hürden entgegen: Während die bei Nominalreduplikation bzw. beim Resultativum vorliegenden syntaktischen Muster relativ klar identifizierbar sind, stellt sich die Syntax von FVG 
strukturell heterogener dar. Es gibt sowohl transitive als auch ditransitive FVG (Berücksichtigung finden, jmdm. eine Antwort geben) sowie Fügungen mit einer PP (zur Aufführung bringen). Dennoch liegen deutlich erkennbare Übereinstimmungen zwischen den genannten FVG-Untertypen vor, die es durchaus als angebracht erscheinen lassen, von einer gemeinsamen Kategorie zu sprechen: Das Nomen ist stets ein Ereignisnomen und dementsprechend häufig durch Derivationsmorpheme (etwa -ung) gekennzeichnet, und beim Verb handelt es sich typischerweise um ein non-manner-Verb (Winhart 2005: 113). Somit ließe sich mit der Struktur in (25) durchaus eine übergreifende schematische Konstruktion postulieren:

$$
\begin{array}{ll}
\text { Sem } & \text { Ereigniskonzept }_{\mathrm{i}}\left(\text { Agens }_{\mathrm{j}} \text {, Patiens }_{\mathrm{k}} / \text { Rezipient }_{\mathrm{m}}\right) \\
\text { Prag } & \text { Figur }_{\mathrm{i}} \text { Vs. Grund }_{\mathrm{n}} \\
\text { Syn } & \mathrm{NP}_{\mathrm{j}} \mathrm{V}_{\mathrm{n} / \text { non-manner }}\left[\mathrm{NP}_{\mathrm{m}}\right] \mathrm{XP}_{\mathrm{i} / \text { Ereignis }} \\
\text { Phon } & \mathrm{Wd}_{\mathrm{j}} \mathrm{Wd}_{\mathrm{n}} \mathrm{Wd}_{\mathrm{k}} / \mathrm{Wd}_{\mathrm{m}} \mathrm{Wd}_{\mathrm{i}}
\end{array}
$$

Die syntaktische Variabilität des Syn-Ansatzes - bei geben-FVG liegt statt eines Patiens ein Rezipient vor - ist zumindest insofern unproblematisch, als in der Konstruktionsgrammatik in erster Linie die gemeinsame Bedeutung und weniger die Form über den Status als Konstruktion entscheidet. So ist für die Resultativkonstruktion vorwiegend das Gegebensein der semantischen Struktur X CAUSE Y BECOME Z BY P ausschlaggebend. Dass dieser Bedeutung sowohl die syntaktische Verbindung NP V NP AP als auch NP V NP PP zugeordnet wird, ist demgegenüber zweitrangig. Wenn man, wie in Kapitel 7.3.2 vorgeschlagen, den implikativen Bedeutungsaufbau bzw. die Herstellung eines Figur-Grund-Effekts als gemeinsames Charakteristikum aller FVG ansieht, wäre damit ein klar identifizierbarer Inhalt für die schematische Konstruktion (25) gegeben.

Hinsichtlich der syntaktischen Struktur der Konstruktion könnte freilich eingewendet werden, dass die Struktur in (25) nicht spezifisch genug ist, wenn man von den Subskripten „non-manner“ und „Ereignis“ absieht. Immerhin können auch Sätze, die nicht die Kriterien für ein FVG erfüllen, diese Struktur aufweisen, vgl. (26).

(26) Der Schauspieler brachte seine Frau zur Aufführung.

Dem steht allerdings entgegen, dass die Struktur dieses Satzes nur oberflächlich mit der eines FVG übereinstimmt, da im letzten Fall zur Aufführung kein lokales Adjunkt ist. In diesem Sinne argumentieren übrigens auch Goldberg/Jackendoff (2004: 536) für die Resultativkonstruktion: Eine Äußerung wie engl. She handed 
him the towel wet entspricht zwar an der Oberfläche der Struktur [NP V NP AP], die für Resultativa kennzeichnend ist. Da jedoch Unterschiede in der syntaktischen Struktur - die AP ist kein Argument, sondern Adjunkt - sowie in der Semantik der AP vorliegen, stellt eine solche bloß oberflächliche Strukturentsprechung keinen Einwand gegen den Ansatz einer Resultativkonstruktion dar. Dies wird man analog auch für die syntaktische Struktur von FVG anzunehmen haben.

Netzwerksemantisch betrachtet stehen FVG damit nicht nur in Vererbungsrelationen, wie sie in Abschnitt 2 beschrieben worden sind, sondern auch in einer Typenhierarchie von über- bzw. untergeordneten Konstruktionen (vgl. Ackerman/Webelhuth 1998: 6; Webelhuth 2011: 154). Ein konkretes FVG erbt somit sowohl von den Teilen, aus denen es zusammengesetzt ist, als auch von dem übergeordneten Typ. Damit konstituiert sich das Netzwerk, in das FVG eingebunden sind, aus ,subpart links“ und ,instance links“ (in der Terminologie von Goldberg 1995: 78f.), vgl. Abb. 9.

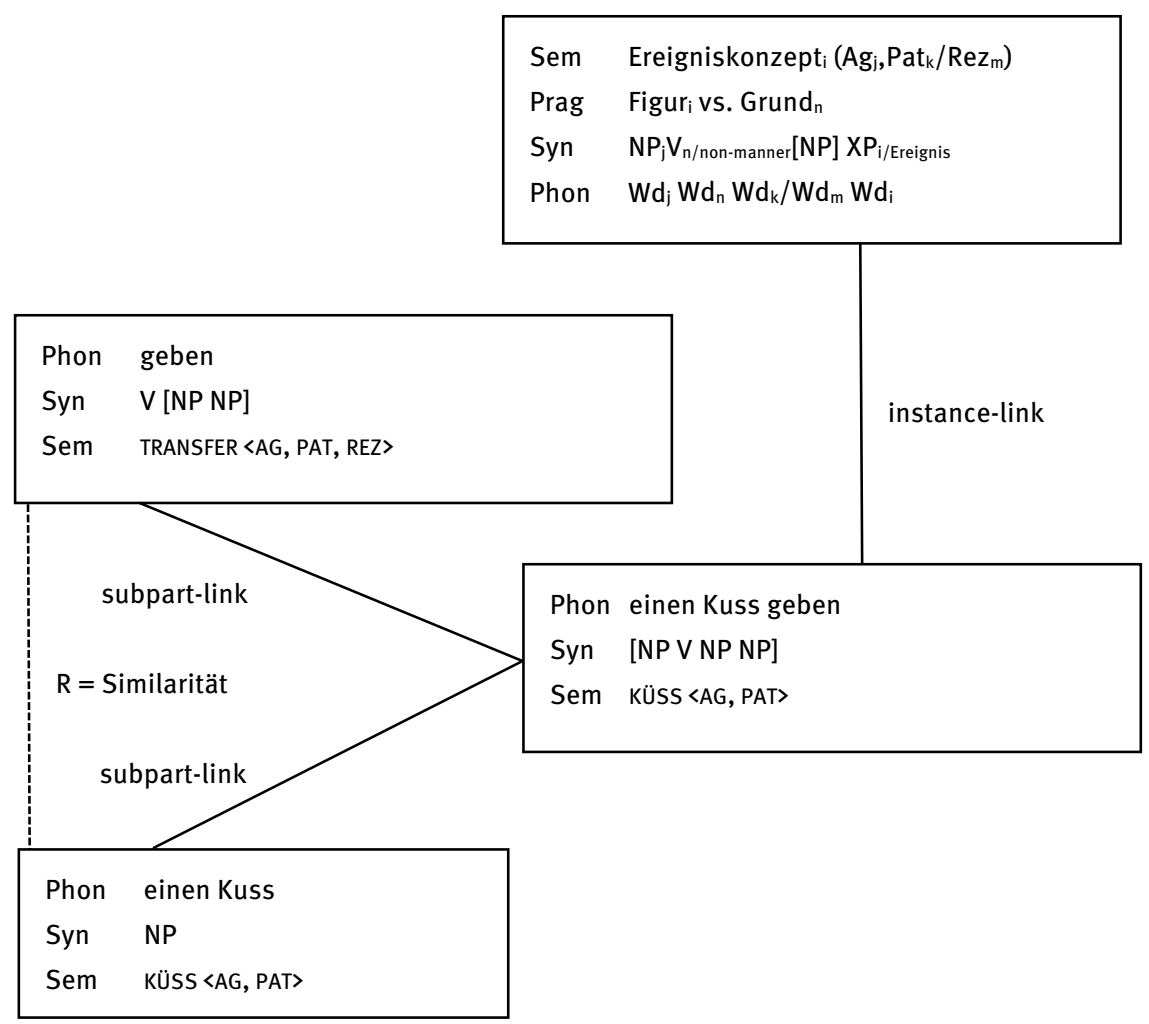

Abb. 9: FVG im Konstruktionsnetzwerk (mit Subpart- und Instance-Links) 


\subsection{Nochmals: FVG als Konstruktionen}

In diesem Kapitel sind FVG aus der Sicht der Konstruktionsgrammatik betrachtet worden. Dabei hat sich vor allem das Konzept der Konstruktionsfamilie, in der idiomatische wie produktive Vertreter der Kategorie FVG koexistieren und in gleicher Weise beschreibbar sind, als hilfreich erwiesen. Auch der Netzwerkmodellierung, die zu den grundlegenden Verfahren der Konstruktionsgrammatik gehört, sind wesentliche Einsichten in Aufbau und Funktionsweise dieser Konstruktionen zu verdanken. Die in Abschnitt 2 erfolgte Klärung der Art und Weise, wie FVG vernetzt sind, hat aber gleichzeitig zu der Erkenntnis geführt, dass es sich bei FVG nur bedingt um eine irreguläre Erscheinung handelt, sondern dass der Aufbau der Konstruktion aus einer Nominalisierung und einem semantisch leeren Verb - und damit auch der pragmatische Gehalt der Verbindung - weitgehend vorhersagbar ist. Dies sei hier noch einmal verdeutlicht: Dass ein Verb semantisch leer ist, stellt zwar insofern eine Irregularität dar, als Verben normalerweise Prädikatsausdrücke sind und als solche einen konzeptuellen Gehalt besitzen. Die Irregularität eines semantisch leeren Verbs kommt jedoch auf erwartbare und reguläre Weise zustande, wie Abb. 8 und 9 veranschaulichen: Dass die Vererbung der semantischen Verbinformation blockiert wird, verstößt in keiner Weise gegen den Grundsatz „Alles wird vererbt“, da die semantische Information des Verbs nicht verloren geht, sondern vom semantisch reichhaltigeren Nomen stellvertretend weitergegeben wird.

Als irregulär kann lediglich eine einzige Erscheinung angesehen werden, nämlich die Verbindung eines Ereignisnomens mit einem Verb, das mit diesem in einer Similaritätsrelation steht. Similarität ist prinzipiell eine paradigmatische Relation, d. h. eine Relation, die miteinander austauschbare Elemente gleicher Art betrifft, welche prinzipiell ein und dieselbe Position in einer Äußerungskette besetzen können; zwischen den Elementen einer Äußerungskette besteht üblicherweise keine Similarität (vgl. Jakobson 1974; Raible 1981/2011: 15), ${ }^{192}$ da die Gefahr der Tautologie besteht. Bei FVG betrifft die Similarität aber gerade Elemente in der Verkettung, es liegt somit eine nicht-kanonische Verkettung von

192 Dazu sei die Stelle bei Raible (1981/2011: 15) ausführlicher zitiert: „Mit seinem genialen Blick für das Grundlegende hat Roman Jakobson [...] die Aufmerksamkeit auf das Zusammenwirken zweier Prozesse beim Sprechen gelenkt: eines paradigmatischen, bei dem Einheiten aus anderen, gleichrangigen Einheiten ausgewählt, und eines syntagmatischen, bei dem die ausgewählten Einheiten in eine lineare Abfolge gebracht werden. Im paradigmatischen Prozess spielt die Dimension ,Similarität - Kontrast‘, im syntagmatischen die Dimension ,Kontiguität - Distanz‘ eine dominierende Rolle“. 
Konstituenten vor. Syntagmatische Similarität bzw. Identität findet sich nur gelegentlich, und zwar interessanterweise ausschließlich bei Strukturen, die als irregulär angesehen werden, etwa bei der oben mehrfach angesprochenen NPNKonstruktion sowie der „salad-salad“-Konstruktion der englischen Umgangssprache (Ghomeshi et al. 2004) (aus dem Umfeld der FVG vgl. auch die in Kapitel 3.3.6 behandelte figura etymologica). Das Besondere, das Idiosynkratische und (wenn man so will) die Irregularität von FVG besteht somit letztlich nur darin, dass eine bestimmte sprachliche Strategie - die Herstellung von Similarität - hier in einem Bereich angewandt wird, in dem dies nicht üblich ist. Die Annahme, dass FVG mit den sonst geltenden sprachlichen Regeln nicht beschrieben werden könnten, ist somit nicht haltbar. Ein näherer Blick auf die syntaktischen Eigenschaften von FVG (s. Kapitel 6) hat ebenfalls erwiesen, dass für FVG im Wesentlichen keine konstruktionsspezifische Syntax anzunehmen ist. Wenn man von einem Kontinuum zwischen Idiosynkrasie und Regel ausgeht, so wird man FVG aber letztlich näher am Pol ,Regel` als am Gegenpol „Idiosynkrasie‘ zu verorten haben.

Gerade mit dem letzten Punkt steht die hier präsentierte Analyse im Widerspruch zu einer Auffassung, die die Kategorie ,Konstruktion“ weitgehend mit Irregularität gleichsetzt. Diese Gleichsetzung liegt auch der klassischen Definition von Goldberg (1995) zugrunde, in der der Konstruktionscharakter eines Form-Bedeutungs-Paares an dessen mangelnder Ableitbarkeit aus den geltenden Regeln geknüpft ist:

C is a construction iff $_{\text {def }} \mathrm{C}$ is a form meaning pair $\left\langle\mathrm{F}_{\mathrm{i}}, \mathrm{S}_{\mathrm{i}}>\right.$ such that some aspect of $\mathrm{F}_{\mathrm{i}}$, or some aspect of $S_{i}$ is not strictly predictable from C's component parts or from other previously established constructions.

(Goldberg 1995: 4) $)^{193}$

Dass ein FVG nicht auf vorhersagbare Weise aus seinen Teilen hergeleitet werden kann, ist, wie Abb. 8 demonstriert, nicht der Fall. Es lässt sich, wie gesagt, allenfalls argumentieren, dass mit der Vererbungsblockade eine Regularität angewandt wird, die sonst selten zum Tragen kommt und die nur hier - und zwar gerade zur Vermeidung einer irregulären tautologischen Äußerung - aktiviert wird.

Damit kommt dieses Kapitel in gewisser Weise zu einem paradoxen Ergebnis: Mit der Netzwerkdarstellung wurde ein Darstellungsmodus gewählt, der in konstruktionsgrammatisch orientierten Ansätzen geläufig ist. Die Beschreibung von FVG als Knoten in einem Netzwerk hatte aber zu einer Modellierung geführt, die

193 In Goldberg (2006: 5) wird diese Definition dahingehend erweitert, dass auch vorhersagbare Muster als Konstruktionen bezeichnet werden, sofern sie ausreichend frequent sind. 
der theoretischen Präferenz der Konstruktionsgrammatik für die Annahme maximaler Speicherung nur sehr bedingt entspricht. Wenn etwa Goldberg (2003b) für ,light verb'-Konstruktionen des Persischen postuliert, dass die Verbindungen von Nomen und Verb nicht online produziert, sondern stets als Einheiten abgespeichert würden, so ist dies nur teilweise auf die hier untersuchten FVG übertragbar: Lediglich für Fügungen wie eine Entscheidung fällen oder die Flucht ergreifen muss eine Speicherung des gesamten Nomen-Verb-Komplexes angenommen werden; für die in Kapitel 5. untersuchten FVG ist dies jedoch nicht nötig, da hier durchaus online-Prozesse vorausgesetzt werden können.

Dieses Ergebnis ist übrigens auch mit psycholinguistischen Befunden kompatibel. So haben Wittenberg/Piñango (2011) und Wittenberg et al. (2014) für FVG wie jmdm. einen Kuss geben nachgewiesen, dass FVG im Vergleich zu der entsprechenden Vollverbvariante (d. h. hier geben in jmdm. ein Buch geben) einen signifikant höheren „processing load“ beanspruchen. In der hier vorgeschlagenen Netzwerkmodellierung wird für FVG ein Vererbungskonflikt angenommen, der durch eine Blockade der konfligierenden Information gelöst wird. Dass dies mehr Verarbeitungszeit erfordert als die reguläre Vererbung bei jmdm. ein Buch geben, ist erwartbar. Der empirisch nachweisbare höhere Verarbeitungsaufwands bei FVG darf somit als Bestätigung des hier vorgeschlagenen Netzwerkmodells gewertet werden. 